\title{
XVI.
}

\section{Beiträge zur Kenntniss des Kohlehydratstoffwechsels.}

\author{
R. Boehm $\begin{gathered}\text { von } \\ \text { in Dorpat. }\end{gathered}$ F. A. Hoffmann
}

\section{Abhandiung.}

Der Kohlehydratbestand des Körpers der Katze.

In vorliegender Abhandlung haben wir eine Reihe von Erfahrungen tiber den Bestand des Thierkörpers an Kohlehydraten bei ausschliesslicher Fleischnahrung zusammengestellt. Es schien uns zum Verständniss der später zu erörternden Beobachtung des raschen Verschwindens aller Kohlehydrate aus dem Körper ${ }^{1}$ ) unerlässlich, eine Vorstellung von den Mengenverhältnissen zu haben, in welchen sich diese Stoffe in einem gegebenen Moment im Körper angehäuft vorfinden können. In wieweit aber die hier vorliegenden Resultate zu Schltissen tiber den Kohlehydratstoffwechsel zu verwerthen sind, dariber soll später discutirt werden.

Versuche uiber den Kohlehydratbestand des Körpers konnten nattirlich nur dann unter einander vergleichbare Ergebnisse liefern, wenn sie an Versuchsthieren von möglichst gleichartiger Ernährung: angestellt wurden. Wir haben uns ausschliesslich der Katze als Versuchsthier bedient. Dieses Thier bietet schon durch seine scharf ausgeprägten Racenmerkmale unschätzbare Vorzlige vor dem Hundegeschlecht. Ausgewachsene Katzen variiren nur in relativ engen Grenzen bezliglich des. Körpergewichts und der Körperoberfläche. Sie lassen sich in grösserer Anzahl leichter verpflegen und reinlich erhalten als Hunde, und nichts ist ungegrtindeter als die Behauptung; dass sie eine längere Gefangenschaft schlecht ertragen.

Unsere Thiere wurden zu je 3-4 Sttiek in gerảumigen Käfigen gehalten und ohne Ausnahme auf reine Fleischkost gesetzt. Die Futterung fand 1-2mal täglich mit frischem, rohem Pferde- oder Rindfleisch statt, und das Futter wurde jedem Thiere einzeln vorgelegt. Thiere, welche zu einem Versuche ausersehen waren, wurden

1) vgl. Centralblatt 1877. No. 27. 
8-10 Tage vorher vou den tibrigen isolirt und täglich vor der Futterung gewogen. Am Versuchstage selbst blieben die Thiere ohne Nahrung.

Um den Kohlehydratbestand einer Katze zu ermitteln, wurde folgendes Verfahren eingeschlagen: Das Thier wurde auf ein Operationsbrett gebunden, die Arteria carotis einer Seite mit einer Kanüle versehen und daraus zunächst in einem tarirten Gefässe Blut zur Feststellung seines Zuckergehaltes entnommen. Hieranf liessen wir das Thier ungesäumt vollends verbluten, öffneten sofort möglichst rasch die Bauchhộle und schnitten die von der Gallenblase befreite Leber heraus. Dieses Organ wurde dann in einer tarirten Schale abgewogen, in bereit stehendes kochendes Wasser gebracht und dann weiter auf Glykogen und Zucker verarbeitet. Endlich wurden noch so rasch als möglich ca. 200-300 Grm. Muskelfleisch. von verschiedenen Körpertheilen abpräparirt, ebenfalls gewogen und auf Glykogen verarbeitet. In den meisten Fällen beschränkte sich die Untersuchung auf Blut, Leber und Muskeln. Nur in Ausnahmefallen mussten auch die Nieren auf Kohlehydrate verarbeitet werden. ${ }^{1}$ ) Diese Beschrănkung war keine willkïrliche. Wir tuberzeugten uns durch mehrere Versuche, dass in den ibrigen Körperorganen, d. h. Centralnervensystem, Milz, Magen und Darmkanal fur gewöhnlich keine wägbaren Mengen von Kohlehydraten enthalten sind.

Der Besprechung der gewonnenen Resultate müssen wir einige Bemerkungen über die von uns benutzten Methoden rorausschicken. Da es sich meist um allbekannte Dinge handelt, so werden wir nur da, wo es unumgänglich nöthig ist, auf die Arbeiten anderer Autoren Bezug nehmen.

\section{\$1. Untersuchung des Blutes.}

Die verarbeiteten Blutmengen betrugen $10-60$ Glm. frischen Blutes, das immer in einem bedeckten Gefäss auf die zweite Decimale genau abgewogen wurde. Wir entnahmen es, wie bereits erwähnt, meist der Carotis, seltener dem Herzen selbst. Es wurde nach der Wägung möglichst rasch durch Kochen mit Glaubersalz und Wasser nach der frther von Claude Bernard angegebenen Methode enteiweisst. Zur Erzielung eines vollig klaren und eiweiss-

1) Es sind das diejenigen Versuche, in welchen den Thieren einige Stunden yor dem Tode Glykogen ins Blut injicirt wurde. Man findet dann unter bestimmten Bedingungen im Nierendecoct beträchtliche Mengen von Zucker. Es wird in einer der spatteren Abhandlungen von diesen Versuchen ausführlicher die Rede sein. 
freien Decoctes ist es vor Allem nöthig; das Blut mit dem Glaubersalz und dem Wasser längere Zeit bei starkem Aufwallen im Kochen zu erhalten. Kurz bevor wir es aufs Filter brachten, setzten wir einige Tropfen Essigsäure hinzu. Zur völligen Erschöpfung des Zuckers fanden wir dreimaliges Extrahiren der fein zerquetschten Coagula mit erneuten Mengen kochenden Wassers ausreichend. Die gesammelten gut gemischten Decocte wurden sofort auf Zucker nach derFehling'schen Methode titrirt und zwar so, dass man die zuckerhaltige Fltissigkeit aus einer Quetschhahnbitrette $\mathrm{zu}$ der in einem Erlenmeyer'schen Kölbchen im Kochen erhaltenen Fehling'schen. Lösung zufliessen liess. Die Titrirung wurde nur dann als befriedigend betrachtet, wenn sich rothe Flocken aus einer klaren Flüssigkeit am Boden absetzten; in triben Flüssigkeiten ist das Versehwinden des blaten Farbentons meist nicht mit der nothwendigen Sicherheit zu beobachten.

Der einzige Uebelstand dieses Verfahrens besteht unseres Eirachtens darin, dass man nux 1,0 höchstens 2,0 C.-Ctm. Fehling'scher Lösung zu einer Titrirung verwenden kann, wodurch eine starke Multiplication der unvermeidlichen Fehler bedingt wird. Es scheint uns daher auch illusorisch, die Bestimmungen itber die zweite Decimale hinaus zu berechnen. Im Uebrigen wird Jeder, der sich mit dieser Methode eingehender beschäftigt, zu der Ueberzeugung: gelangen, dass sie allen stellbaren Anfordertungen vollkommen entspricht.

Den Zuckergehalt des Gesammtblutes erhielten wir, indem wir die Menge des Gesammtblntes zu 1/13 des Körpergewichts annahmen. Dabei wird selbstverständlich die Verschiedenheit des Zuckergehaltes des Blutes in verschiedenen Gefässprovinzen vernachlässigt, was wir ohne Bedenken thun konnten, da es uns nur auf Annäherungswerthe fur den Gesammtblutzuckergehalt ankam. Ausser der Enteiweissungsmethode durch Kochen mit Glaubersalz haben wir auch mehrfach Versuche mit der gleichfalls von Bernard angegebenen Methode des Enteiweissens durch Kochen des Blutes mit Thierkohle angestellt. Dieses Verfahren ist zeitraubender, liefert aber ebenfalls befriedigende Resultate; nur muss die Kohle mindestens 10-12 mal bis zur völligen Erschöpfung des Zuckers mit kochendem Wasser extrahirt werden. Die nachstehenden Versuchsdaten zeigen, dass beide Methoden gut übereinstimmende Resultate ergeben.

Versuch C. Kater von 3,06 Kilo. a) 17,56 Grm. Blut, aus der Carotis entnommen, mit Glaubersalz enteiweisst, geben 240,0 C. - Ctm. Decoct; die Titrirung ergibt $\mathbf{0 , 2 3}$ pCt. Zucker. b) 12,16 Grm. Blut aus 
der Carotis desselben Thieres, unmittelbar nach dem ersten Aderlass entnommen, gaben mit Thierkohle enteiweisst 294,0 C.-Ctm. Decoct. Die Titrinung ergibt 0,24 pCt. Zucker.

Da, wo es sich nur um qualitative Zuckerbestimmungen im Blute handelte, hahen wir das Blutdecoct längere Zeit (10 Minuten bis $1 / 4$ Stunde) in einer Porzellauschale mit kleinen Mengen Fehling'scher Losung im Kochen erhalten. Erfolgte hierbei keine Ausscheidung von Kupferoxyclul, welche häufig in Form eines schỏn orangerothen Beschlages der Schalenwand auftritt, so betrachteten wir das Blut als zuckerfiei. Eine schmutzig'-gelbliche, flockige Ausscheidung, die sich nach dem Erkalten des laugere Zeit gekochten Gemisches bisweilen einstellte, liessen wir niemals als Zuckerieaction gelten, da wir uns zur Genige davon itberzeugt hatten, dass auch minimale Zuckermengen im Blutdecoct schon während des Kochens mit der Fehling'schen Lösung eine schön orangerothe Kupferoxydulausscheidung bewirken, die namentlich auf dem weissen Grunde der Porzellanschale nicht ibersehen werden kam.

In neuester Zeit hat $\mathrm{Cl}$. Bernard ${ }^{1}$ ) mit besonderem Nachdruck darauf aufmerksam gemacht, dass sich der Zucker in dem aus der Ader gelassenen Blute sowohl als anch im Blute todter Thiere und Menschen ungemeiu rasch zersetze, so dass blos diejenigen Versuche über den wirklichen Zuckergehalt des Blutes richtigen Aufschluss geben sollen, welche ummittelbar nach dem Aderlass am lebenden Thiere mit möglicbst frischem Blute ausgefuhrt werden. Er theilt mehrere Zahlenreihen mit, aus denen hervorgeht, dass nach lingerer Aufbewahrung bei Sommertemperatur das Blut von Hunden so schnell an Zuckergehalt abmimmt, dass nach 24 Stunden der Zucker aus dem Blute vollig verschwunden sein kann. Die Behauptung, dass anch innerhalb des Körpers nach dem Tode des Thieres eine gleich rasche Zersetzung des Blutzuckers stattfindet und dass daher Zuckerbestimmungen, welche sich anf das längere Zeit nach dem Tode des Thieres dem Gefasssystem entnommene Blut beziehen, nichts werth sind, wird nicht näher durch Versuchsdaten belegt. Wir müssen diese Frage hier kurz berühren, weil vielleicht auf Grund der Bernard'schen Aufstellungen einige wenige der in den folgenden Abhandlungen mitgetheilten Versuche als unbrauchbar erscheinen könnten. Der Leser wird bei der Durchsicht der Protokolle bemerken, dass iiberall die Zeit der Untersuchung nach dem Tode oder nach dem Aderlass reveichnet ist, und

1) Journ. de Pharmacie et de Chimie IV. Ser. T. XXIV. Aug.-Sept. 1876. 
dass in der grossen Mehrzahl der Fälle die chemische Untersuchung unmittelbar nach dem Tode oder nach dem Aderlass begonnen wurde. Un dies zu ermöglichen, haben wir in vielen Versuchen die Beobachtungen auch während der Nacht nicht unterbrochen; trotzdem konnten wir es nicht verhindern, dass in einigen Fallen die chemische Untersuchung des Thieres erst Stunden nach dem Tode desselben begonnen wurde. Es handelte sich auch in diesen Versuchen, von denen in späteren Capiteln ausfiubrlicher die Rede sein vird, lediglich um die Frag'e: enthalten die Organe des Thieres noch Kohlehydrate oder nicht? Das Resultat war in der Regel der völlige Mangel aller Kohlehydrate in Blut, Leber und Muskeln. Sind num diejenigen Versuche, wo die Analyse erst mehrere Stunden nach dem Tode begann, auf Grund der Angaben von Cl. Bernard zu verwerfen? Wir durften nicht a priori annehmen, dass der Zueker, in Blute des todten Thieres unverändert bleibt. Es konnten dariber nur specielle Versuche Aufschluss geben.

Den Ergebnissen derselben ist kurz vorauszuschicken, dass 1. unsere Experimente nicht im Sommer sondern bei streng'er Winterkälte und einer Zimmertemperatur von höchstens $18^{\circ} \mathrm{C}$. angestellt wurden; dass 2. in mehreren der hierhergehörigen Fälle das Thier unmittelbar nach dem Tode in einen kalten Raum gebracht wurde; dass 3. alle unsere Thiere bei einer abnorm niedrigen Innentemperatur von $30-25^{\circ}$ C. starben; und dass 4. die Analyse nur ein einziges Mal 9 Stunden, in den übrigen Fällen $1 / 2$ bis hüchstens 6 Stunden nach dem Tode begann. Dass nun in der That unter solchen Bedingangen eine Zersetzung des Blutzuckers nicht stattfindet, zeig'en nachfolgende Versuchsdaten.

Wir tödteten zwei Katzen durch Strangulation und liessen beide Leichen bei einer Zimmertemperatur von $18^{\circ} \mathrm{C}$. auf dem Tische liegen. Der einen todten Katze wurde nach 3 Stunden, der anderen nach 6 Stunden Blut aus dem Herzen zur quantitativen Zuckerbestimmung entnommen. Diese beiden Fälle wurden mit einem dritten verglichen, wo das Blut einer erdrosselten Katze unmittelbar nach dem Tode aus dem Herzen entnommen und untersucht wurde.

I. Blut 3 Stunden nach dem Tode $(13,95 \mathrm{Grm}$.) enthält $\mathbf{0 , 3 2}$ pCt. Zucker.

II. Blut 6 Stunden nach dem Tode $(11,77$ Grm.) enthält $0,30 \mathrm{pCt}$. Zucker.

III. Blut unmittelbar nach dem Tode $(38,39 \mathrm{Gr}$.) enthält 0,21 pCt. Zucker.

Wix verzichten für jetzt auf eine nähere Untersuchung des un- 
erwarteten Befundes, dass der Blutzuckergéhalt nach dem Tode innerhalb 6 Stunden nicht ab-sondern offenbar zugenommen hatte, glauben aber daraus die Berechtigung entnehmen zu dürfen, auch diejenigen unserer Blutzuckerbestimmungen za verwerthen, welche nicht unmittelbar nach dem Tode des Thieres ausgefuhrt werden kounten. Dass sich der Blutzucker in der That nicht immer so rasch zersetzt, wie es nach Cl. Bernard's in der Sommerzeit angestellten Beobachtungen scheinen könnte, erhellt noch aus folgendem Versuche. Wir verwahrten $112,0 \mathrm{Grm}$. aus der Carotis einer Katze entnommenen Blutes bei der Temperatur eines ungeheizten Raumes während des Decembers (Aussentemperatur -10-0 ${ }^{\circ}$ R.) 3 Wochen lang 'in einem leicht bedeckten Becherglase und bestimmten am Ende der dritten Woche den Zucker; es fand sich noch $\mathbf{0 , 1}$ pCt. Zucker vor.

\section{\$2. Untersuchung der Leber.}

Zur Ermittlung. des Kohlehydratbestandes der Leber waren Zucker- und Glykogenbestimmungen erforderlich. Erstere hätte man nur dadurch umgehen können, dass man nach der Vorschrift von Pavy, Meissner u. A. die Leber unmittelbar aus dem Leib des lebenden Thieres utber kochendem Wasser möglichst schnell entfernt und sofort ins kochende Wasser gebracht hätte.

Dass man bei diesem Verfahren in der That nur etwa so viel Zucker in der Leber findet, als dem darin zuritckgehaltenen Blute entspricht, also minimale, kaum bestimmbare Mengen, können auch wir durch unsere eigene Erfahrung bestätigen. Es mögen die einschlägigen Beobachtungen hier kurz mitgetheilt werden.

Versuch XCrII. Einer 2,2 Kilo schweren Katze, welche seit langerer Zeit ausschliesslich mit Fleisch gefüttert worden war, wird mit einem grossen chirurgischen Amputationsmesser der Bauch aufgeschnitten, die möglichst rasch herausgeschnittene Leber sofort in bereit stehendes kochendes Wasser geworfen, unter demselben mit der Scheere zerkleinert, später zerrieben und mehrmals ausgekocht.

Die erhaltenen $230,0 \mathrm{C}$.Ctm. Leberdecoct enthalten in toto 0,033 Grm. Zucker, also nngefähr soviel als 15,0 Grm. Blut entspricht, welche leicht in der Leber zurückgeblieben, sein kömnen.

Versueh XCIV. Katze von 2,0 Kilo. Leber genau so behandelt, wie im vorigen Versuche; sie enthält keinen Zucker, aber reichliche Mengen Glykogen.

Versuch $\mathbf{X} C V$. Katze von 1,65 Kilo; hat seit 3 Tagen gehungert. Lèber enthält reichlich Glykogen aber keinen Zueker.

Versueh XCVI. Einer krräftigen Katze (gewöhnliche Fütterung mit Pferdefleisch) wird der Bauch über. Kochendem Wasser aufgeschlitzt, so 
dass das Blut aus den durcbschnittenen Gefässen direct ins kochende Wasser fliesst. Die möglichst rasch ausgeschnittene Leber wird sofort in eine zweite bereit stehende Schale mit kochendem Wasser geworfen und in gewöhlicher Weise ausgekocht. Is zeigt sich, dass die bei der Operation vergossene geringe Menge Blut mehr Zucker enthält als die ganze Leber, deren Decoct nur eine minimale Zuckerreaction gibt.

Der Zuckergehalt dermaassen aus dem lebenden Thiere entnommener Lebern hätte also offenbar bei der Feststellung des Kohlehydratbestandes der Leber vernachlässigt werden können. Doch war diese Methode fur unsere Zwecke nicht anwendbar; es verflossen im guinstigsten Falle doch 10-15 Minuten, ehe die Leber ins kochende Wasser kam, ein Zeitraum während dessen sich erhebliche Zuckermengen in der Leber bilden konnten. Die Bestimmung des Leberzuckers war demnach nicht zu umgehen, und wir haben sie nach folgenden Methoden ausgeführt. Die gewogene Leber wurde in kleimen Sticken in kochendes Wasser eingetragen. Nach kurzem Aufwallen wurden die durch das Kochen erhärteten Leberstücke mit einem Hornlöffel aus der Flissigkeit herausgeschöpft und in einem grossen Porzellanmörser zu einem möglichst feinen Brei zerrieben. Dieser Brei nun wurde so lange mit erneuten Mengen destillirten Wassers ausgekocht, bis eine Probe des Filtrates keine Zucker(resp. Glykogen-) reaction mehr gab. Beim erstmaligen Auskochen wird behufs Ausfällung der Eiweisskörper Essigsäure bis zur schwach sauren Reaction zugesetzt. Die Decocte wurden vereinigt, gut gemischt, das Volumen bestimmt und dann in zwei Portionen getheilt, von denen die kleinere von 100,0-200,0 C.-Ctm. zur Zuckerbestimmung, die grössere zur Darstellung des Glykogen diente. Zur Extraction des Zucker's genigt in der Regel 3-4 maliges Auskochen, soll aber auch das Glykogen völlig erschöpft werden; so muss die Extraction oft $10-20$ mal wiederholt werden.

Zur Fällung des Glykogens und Eiweisses aus der zur Zuckerbestimmung dienenden Flüssigkeit versetzten wir dieselbe nach rolausgegangener Neutralisation mit ihrem dreifachen Volumen $95^{\circ} \mathrm{Al}$ kohols, liessen das Gemenge in einem geräumigen Becherglase tiber einer kleinen Spiritusflamme mehrmals aufkochen und filtrirten nach dem Erkalten. ${ }^{1}$ ) Das Filtrat wurde bei saurer Reaction auf dem Wasserbade bis zur völligen Verjagung des Alkohols erwärmt und zum dỉnnen Syrup eingedampft, der mit Wasser verdünnte Ritckstand abermals filtrirt, das Filter gut ausgewaschen und das Filtrat

1) vgl. J. Puls, Ueber quantitative Eiweissbestimmung. Pflüger's Archlv. XIII. 1876. 
wieder genau auf 100,0 C.-Ctm. gebracht, endlich aber so wie das Blutdecoct nach der Fehling'schen Methode titrirt. Man kann hier in der Regel die Titrirung mit 3-5 C.-Ctm. Eehling'scher Lösung: ansfübren und die Endreaction tritt stets mit vollendeter Schärfe ein. Durch einfache Multiplication exhält man den Zuckergehalt der ganzen Leber. Man kann indessen das Leberdecoct, das, wenn die Essigsäure im richtigen Verhältnisse zugesetzt wurde, nur noch Spuren von Eiweiss enthäl, auch entweder direct oder nach vorheriger Behandlung mit Thierkohle zur Titrirung verwenden. Die Anwesenheit von Glykogen in nicht allzu grosser Menge stört die Reaction nicht wesentlich. Geringe Mengen Glyliogen werden ausserdem von der Thierkohle zurickgehalten.

Wo es übrigens auf grössere Genauigkeit ankommt, ist die Methode der Fällung des Eiweiss und Glykog'en durch Alkohol nicht wohl zu umgehen.

Den Glykogengehalt der Leber ermittelten wir, indem wir aus der zweiten grösseren Portion des Decocts nach der Bu $\mathfrak{i} \mathrm{ck}$ e'schen ${ }^{1}$ ) Methode das Glylkogen rein darstellten. Es wurde anf dem Filter bei $100-105^{\circ} \mathrm{C}$. im Luftbad getrocknet, dann gewogen und jedesmal auf seine Reinheit geprift. Eine einfache Berechnung ergab. sodann den Glykogengehalt der ganzen Leber.

Den Gesammtkohlehydratbestand der Leber haben wir erhalten, indem wir die für das Glykogen erhaltene Zahl auf Zucker umrechneten (100,0 Grm. Glykogen entsprechen 111,11 Grrm. Zucker) und Leberzucker und den aus Glykogen berechneten Zucker addirten.

\section{-\$3. Untersuchung dor Muskeln.}

Um den Glykogenbestand der Musculatur zu ermitteln, war es nothwendig; entweder in jedem Einzelversuch sämmtliche Muskeln abzupräpariren und ihr Gewicht zu bestimmen, oder einen aus mehreren Beobachtungen gewonnenen Mittelwerth des Gewichts der Gesammimusculatur den einzelnen Bestimmungen zu Grunde zu legen. Wir haben in 10 Versuchen das Gewicht der Gesammatmusculatur direct bestimmt; die gewonnenen Zahlen sind in Tabelle I (S. 279) zusammengestellt.

Fiur die Ermittlung des durchschnittlichen Verhältnisses des Muskelgewichtes zum Körpergewicht eignen sich ganz besonders die ersten $7 \mathrm{der}$ in der Tabelle zusammengestellten Versuche, weil sie vollig gleichmässig ernährte und ad hoc getödtete Thiere betreffen.

1) Wiener Sitzungsbericht, mathem,-physik. Cl, Bd. LXII. 2. S. .1-9. 
Nach denselben beträgt das durchschnittliche Muskelgewicht unserer Thiere 0,38 also annähernd 0,40 des Körpergewichts. Diesen Werth haben wir der Berechnung des Muskelgewichts in einig'en Fällen zu Grunde gelegt, wo dasselbe nicht direct bestimmt worden ist.

Tabelle 1 .

\begin{tabular}{|c|c|c|c|c|c|}
\hline 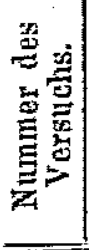 & 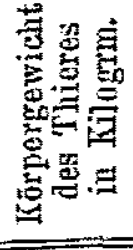 & Axt der Fütterung. & $\begin{array}{l}\text { Gesimint- } \\
\text { gewicht } \\
\text { der } \\
\text { abppriparirten } \\
\text { Muskeln } \\
\text { in Grm. }\end{array}$ & $\begin{array}{c}\text { Köryer- } \\
\text { gewicht } \\
\text { dividirt durch } \\
\text { STuskelgewicht. }\end{array}$ & $\begin{array}{c}\text { Todesart, } \\
\text { Versuelsanording }\end{array}$ \\
\hline 131 & 3,22 & Rindfleisch. & 1210,0 & 0,37 & $\begin{array}{l}\text { ad hoc durch Verblu- } \\
\text { tung gotödtet. }\end{array}$ \\
\hline 132 & 3,02 & Rindfleisch. & 1160,0 & 0,39 & desgl. \\
\hline 133 & 2,60 & Rindfleisch. & 1017,0 & 0,40 & desgl. \\
\hline 134 & 2,79 & Rindfleisch. & 1130,0 & 0,40 & desgl. \\
\hline 135 & 2,38 & Rindfleisch. & 908,0 & 0,38 & desgl. \\
\hline 76 & 3,12 & Pferdefleisch, & 1204,0 & 0,38 & desgl. \\
\hline 71 & 2,27 & Milch und Fleisch. & 803,5 & 0,35 & desgl. \\
\hline 105 & 2,72 & Fleisch. & 931,0 & 0,34 & $\begin{array}{l}\text { Fesslungsversuch; stirbt } \\
\text { mach } 24 \text { Stunden. }\end{array}$ \\
\hline 74 & 3,21 & Fische. & 1133,0 & 0,35 & $\begin{array}{l}\text { Rückenmarksdarchschnoi- } \\
\text { dung, stirbt aufd. Ope- } \\
\text { rationsbrett. }\end{array}$ \\
\hline 72 & 2,60 & gemischteNahrung. & 1151,0 & 0,44 & Fesslungsversuch. \\
\hline
\end{tabular}

Zur Glylogenbestimmung wurden nun in jedem einzelnen Falle möglichst rasch nach dem Tode des Thieres von verschiedenen Körperpartien desselben ca. 200-300,0 Grm. Muskeln möglichst frei von Sebnen und Fett abpräparirt, in der Fleischhackmaschine zerlsleinert, genau gewogen und hierauf ebenso wie die Leber auf Glykogen verarbeitet. Nach längerem energischem Kochen wird das Fleisch so weich, dass man es bequem in einem Porzellanmörser zu einem feinen Brei zerreiben kann, aus welchem dann nach Fällung des Eiweisses durch Essigsäure das Glykogen durch 4-6 maliges Auskochen mit erneuten Wassermengen völlig extrahirt werden kann. Die ungemein empfindliche Jodreaction diente als Anhaltspunkt fur die vollständige Erschöpfung des Muskelglykogens. Aus den gesammelten Decocten wird das Glykogen nach der Brifeke'schen Methode rein dargestellt, getrocknet, gewogen und ftir die Gesammtmusculatur berech net.

\$ 4. Ergebnisse der Versuche über den Kohlehydratbestand.

Der Anhang zu dem vorliegenden Abschnitt enthalt die Protokolle von 20 Versuchen, welche von uns zur Ermittlung des Kohlehydratbestandes des Katzenkörpers angestellt worden sind. In 6 dieser Versuche erstreckte sich die Untersuchung anf Blut, Leber und 
Muskeln, in 2 blos auf Blut und Leber, in 3 nur auf das Glykogen der Leber, in 10 nur auf den Zuckergehalt des Blutes und der Leber. Zur leichteren Uebersicht sind die Resultate in Tabellen zusammengestellt, zu welchen hier einige Bemerkungen gefugt werden sollen.

Tabelle II. enthält die Zusammenstellung von 26 Blutzuckerbestimmungen, von denen die meisten das aus der Carotis entnommene Blut wohlgenährter Katzen betreffen; 3 davon beziehen sich auf Hungerthiere.

Tabelle II.

\begin{tabular}{|c|c|c|c|c|c|}
\hline 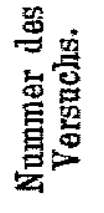 & $\begin{array}{l}\text { Körpergewicht } \\
\text { in Kilogramm. }\end{array}$ & $\begin{array}{l}\text { Untersuchtes } \\
\text { Blut } \\
\text { in Grm. }\end{array}$ & $\begin{array}{l}\text { Zuckergehalt } \\
\text { des Blutes } \\
\text { in pCt. }\end{array}$ & $\begin{array}{c}\text { Zuckergehalt } \\
\text { des } \\
\text { Gesammtulutes. }\end{array}$ & Bemerkungen. \\
\hline $\begin{array}{r}49 \\
50 \\
51 \\
52 \\
55 \\
57 \\
59 \\
60 \\
61 \\
62 \\
68 \\
71 \\
76 \\
85 \\
86 \\
98 \\
100 \\
102 \\
103 \\
134 \\
130 \\
135 \\
139 \\
147 \\
53 \\
54\end{array}$ & $\begin{array}{l}1,37 \\
2,0 \\
3,87 \\
2,76 \\
3,25 \\
2,50 \\
3,13 \\
2,95 \\
3,30 \\
2,74 \\
2,72 \\
2,27 \\
3,12 \\
2,72 \\
2,77 \\
3,01 \\
3,06 \\
3,02 \\
2,65 \\
2,79 \\
4,07 \\
2,38 \\
2,38 \\
2,65 \\
1,65 \\
1,68\end{array}$ & $\begin{array}{l}30,96 \\
42,05 \\
62,12 \\
34,12 \\
29,27 \\
11,13 \\
24,80 \\
18,36 \\
29,70 \\
25,66 \\
35,59 \\
38,27 \\
43,17 \\
17,87 \\
15,63 \\
29,64 \\
14,52 \\
22,78 \\
35,09 \\
25,58 \\
26,12 \\
25,38 \\
38,39 \\
28,32 \\
25,79 \\
37,22\end{array}$ & $\begin{array}{l}0,13 \\
0,13 \\
0,13 \\
0,13 \\
0,13 \\
0,12 \\
0,15 \\
0,17 \\
0,12 \\
0,13 \\
0,13 \\
0,12 \\
0,13 \\
0,13 \\
0,13 \\
0,20 \\
0,20 \\
0,31 \\
0,19 \\
0,21 \\
0,26 \\
0,21 \\
0,21 \\
0,11 \\
0,18 \\
0,13\end{array}$ & $\begin{array}{l}0,136 \\
0,199 \\
0,391 \\
0,273 \\
0,325 \\
0,228 \\
0,360 \\
0,374 \\
0,312 \\
0,281 \\
0,277 \\
0,244 \\
0,331 \\
0,280 \\
0,279 \\
0,46 \\
0,46 \\
0,71 \\
0,38 \\
0,44 \\
0,80 \\
0,378 \\
0,378 \\
0,261 \\
0,165\end{array}$ & $\begin{array}{l}8 \text { Tage Hunger. } \\
3 \text { Tage Hunger. } \\
3 \text { Tage Hunger. }\end{array}$ \\
\hline
\end{tabular}

Wir ersehen aus den letzten 3 Versuchen, dass, während eine dreitägige Hungerperiode keine deutliche Verminderuag des Blutzuckergehaltes zu bewirken vermag, eine solche nach 8 tägigem Hunger bereits unverkennbar zu Tage tritt. Das Blut mehrerer Katzen, welche den Hungertod gestorben waren, fanden wir ganz zuckerfrei. In 18 Versuchsnummern (den ersten 16 und den beiden Hungerversuchen No. 53 und 54) schwankt der Procentgehalt des Blutes an Zucker zwischen $0,12-0,18 ; 11$ mal finden wir die Zahl 
$0,13,4 \mathrm{mal} 0,12$ und nur $3 \mathrm{mal}$ höhere Werthe von $0,15,0,17$ und 0,18 . In den 8 übrigen Versuchen schwankt der Znckergehalt des Blutes zwischen $0,19-0,31$. Die ersten 18 Versuche, aus welchen sich ein Durchschnittswerth von 0,13 ergibt, waren von November 1875 bis Mai 1876 ausgefuhrt worden, während die zweite Gruppe mit dem Durchschnittswerthe von $0,20 \mathrm{pCt}$. aus den Monaten October 1876 bis Februar 1877 stammt. Dass nun dieser immerhin bemerkenswerthe Unterschied nicht etwa auf einem verschiedenen Titre unserer Fehling'schen Lösungen beruhte, davon haben wir uns sicher iiberzeugt; und da wir auch beim Titriren stets in gleicher Weise verfuhren, so missen wir wohl annehmen, dass die Katzen der zweiten Periode ein zuckerreicheres Blut ftihrten als die der ersten. Es ist uns nicht unwahrscheinlich, dass dieser Unterschied durch die reichlichere Fütterung der Katzen der zweiten Periode bedingt war. Der Durchschnitt aus allen Bestimmungen des Blutzuckergehalts beläuft sich auf $0,15 \mathrm{pCt}$, eine $\mathrm{Zahl}$, welche mit den von anderen Autoren erhaltenen Werthen, namentlich aber den von Cl. Bernard (a. a.: 0.) neuerdings gelieferten Daten gut itbereinstimmt. Lässt sich somit aus Tabelle II unzweifelhaft die annähernde Constanz des Blutzuckergehaltes erkeñnen, die man nach den bereits rorliegenden Erfahrungen sicher erwarten durfte, so sieht man in Tabelle III, (s. S. 282), welche die Zusammenstellung von 29 Leberzuckerbestimmungen normaler Thiere enthält, ziemlich erhebliche Schwankungen. Columne 5 enthält die Werthe, welche den Procentgehalt der frischen Leber an Zucker angeben, Columne 6 das Verhältniss des Leberzucker's zum Körpergewicht.

Man ersieht aus Columne 6 dieser Tabelle, dass die Zahl, welche das Verhältniss des Leberzuckers zum Körpergewicht angibt, in einer grossen Anzahl von Einzelbeobachtungen, einen annälernd ubereinstimmenden Werth von $0,5-0,6$ repräsentirt. Wir wurden dadurch zu der Vermuthung gedrängt, es konnte vielleicht doch nur ein beschränkter Antheil des in der Leber aufgespeicherten Glykogens postmortal in Zucker tibergehen, eine Menge, welche möglicherweise in einem festen Verhältnisse zum Körpergewicht der Thiere stand. Allerdings widersprachen dieser Annahme von volneherein die älteren Angaben Cl. Bernard's und Schiff's. Nichtsdestoweniger haben wir einige Versuche mit Hinsicht auf dieses Verhalten angestellt. War unsere Vermuthung eine irrige, so gab es fuir die scheinbare Constanz des Leberzuckergehaltes nur die eine allerding's sehr einfache Erklärung, dass, da wir alle Lebern in ziemlich gleichen Zeitpunkten nach dem Tode des Thieres untersuchten, in diesen Zeit- 
räumen im Verhältniss zum Körpergewicht des Thieres annähernd gleiche Glykogenmengen in Zucker tibergingen.

Tabelle III.

\begin{tabular}{|c|c|c|c|c|c|}
\hline 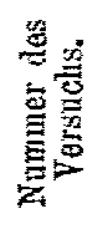 & $\begin{array}{l}\text { Iöppergewicht } \\
\text { in filostumm }\end{array}$ & $\begin{array}{l}\text { Gewicht } \\
\text { dor } \\
\text { frischen Leber } \\
\text { in Gr'm. }\end{array}$ & $\begin{array}{c}\text { Zuckergehalt } \\
\text { der } \\
\text { Leber } \\
\text { in Grun }\end{array}$ & $\begin{array}{l}\text { Zuekergehalt } \\
\text { in pCt, } \\
\text { des Gewichts } \\
\text { dor frischon } \\
\text { Leber. }\end{array}$ & $\begin{array}{c}\text { Leberzucker } \\
\text { pro I Kilo } \\
\text { Katze. }\end{array}$ \\
\hline $\begin{array}{l}50 \\
51 \\
52 \\
53 \\
54 \\
55 \\
56 \\
57 \\
59 \\
60 \\
61 \\
62 \\
65 \\
68 \\
71 \\
76 \\
87 \\
86 \\
102 \\
100 \\
140 \\
142 \\
143 \\
130 \\
131 \\
132 \\
133 \\
134 \\
135\end{array}$ & $\begin{array}{l}2,0 \\
3,87 \\
2,76 \\
1,65 \\
1,68 \\
3,25 \\
3,53 \\
2,50 \\
3,13 \\
2,95 \\
3,30 \\
2,74 \\
3,36 \\
2,72 \\
2,27 \\
3,12 \\
2,07 \\
2,77 \\
3,02 \\
3,06 \\
4,07 \\
2,55 \\
2,20 \\
4,07 \\
3,22 \\
3,02 \\
2,60 \\
2,79 \\
2,38\end{array}$ & $\begin{array}{r}\bar{Z} \\
\bar{Z} \\
128,6 \\
118,0 \\
- \\
- \\
- \\
- \\
87,1 \\
59,3 \\
127,1 \\
72,0 \\
100,0 \\
60,5 \\
90,5 \\
107,0 \\
83,0 \\
82,7 \\
205,0 \\
146,0 \\
126,0 \\
154,0 \\
80,4 \\
75,0\end{array}$ & $\begin{array}{l}0,78 \\
1,98 \\
1,42 \\
0,76 \\
0,39 \\
1,78 \\
2,00 \\
1,40 \\
1,96 \\
1,80 \\
1,95 \\
1,77 \\
2,16 \\
1,28 \\
0,64 \\
1,74 \\
1,03 \\
1,38 \\
1,53 \\
1,51 \\
1,32 \\
1,06 \\
1,10 \\
2,48 \\
1,22 \\
1,98 \\
1,65 \\
1,73 \\
1,36\end{array}$ & $\begin{array}{l}\text { - } \\
\overline{-} \\
\overline{1} \\
1,3 \\
1,6 \\
- \\
- \\
- \\
- \\
1,4 \\
1,0 \\
1,3 \\
1,5 \\
1,3 \\
2,5 \\
1,5 \\
1,1 \\
1,2 \\
1,3 \\
1,3 \\
0,8 \\
1,5 \\
1,0 \\
2,1 \\
1,8\end{array}$ & $\begin{array}{l}0,39 \\
0,51 \\
0,51 \\
0,46 \\
0,23 \\
0,54 \\
0,56 \\
0,56 \\
0,62 \\
0,61 \\
0,56 \\
0,64 \\
0,64 \\
0,47 \\
0,24 \\
0,55 \\
0,49 \\
0,49 \\
0,56 \\
0,46 \\
0,32 \\
0,41 \\
0,50 \\
0,69 \\
0,39 \\
0,65 \\
0,63 \\
0,62 \\
0,57\end{array}$ \\
\hline
\end{tabular}

Die experimentelle Entscheidung war sehr einfach. Wir untersuchten verschiedene Stücke ein und derselben Leber verschieden lange Zeit nach dem Tode des Thieres quantitativ auf Zucker, wie es bereits Cl. Bernard und M. Schiff') gethan hatten.

Versuch CXXXY. 7/I. 77. Starke, weibliche Katze, seit längerer Zeit ausschliesslich mit frischem Rindfleisch gefüttert. Körpergewicht 2,85 Kilo. Das Thier wird rasch erdrosselt und die Leber ausgeschnitten; sie wiegt nach Entfernung der Gallenblase 146,0 Grm. $=1 / 22$ des Körpergewichts. Die Leber wird nun in zwei Portionen getheilt. Das I. Stück 51,5 Grm. schwer wird sofort in Arbeit genommen und bis zur

1) Untersuchungen über die Zuckerbildung in der Leber. Würzburg 1859. S. 149. Schiff theilt hier zahlreiche quantitative Bestimmungen mit, welche zeigen, wie der Zuckergehalt der Leber beim blossen Liegen derselben sich ändert. Die Versuclie beziehen sich besonders auf Eichhorn- und Kaninchenleber. 
Erschöpfung des Zuckers ausgekocht. Man erbält 310,0 C.-Ctm. Decoct. Davon werden 95,0 C.-Ctm. wit 3 Volum. $95^{\circ}$ Alkohol in einem Becherglase gekocht, das ausgeschiedene Eiweiss und Glykogen abfiltrirt und dis Filtrat nach Verdunstung des Alkohols auf dem Wasserbade auf 100,0 C.-Ctm. gebracht. Daron reduciren 7,5 C.-Ctm. 2,0 C.-Ctm. Fehling'scher Lösung. Das Gesammtdecoct des I. Leberstïcks enthält demnach 0,433 Grm. Zucker, 100,0 Leber 0,84 Grm. Zucker und die ganze Leber 1,226 Grm. Z u cker.

Eine Stunde später wird das II. Stïck der Leber, 94,5 Grm. sohwer, verarbeitet. Man erhält 840,0 Decoct; nach dem Enteiweissen u. s. w. reduciren 5,9 C.-Ctm. 2,0 C.-Ctm. Fehling'scher Lösung. Das Decoct des II. Leberstilcks enthält demnach 1,41 Grm. Zucker, 100,0 Grm. Leber 1,5 Grm., die ganze Leber 2,14 Grm. Zncker.

Der Zuckergehalt der Leber ist demnach innerhalb einer Stunde von $0,48-1,5$ pCt, also um 0,66 pCt. gestiegen.

Versuch CXXXIr. 9/I. 77. Kater von 3,02 Kilo, mit Rindfleisch gefuttert. Leber 126,0 Grm. schwer, in zwei Stücke von 53,0 und 73,0 Grm. sehwer zerschnitten.

I. Stulck, von 53,0 Grm. gibt 670,0 Decoct; nach dem Enteiweissen reduciren 20,0 C.-Ctm. 5,0 C.-Ctm. Fehling'scher Lösung. Demnach enthält das Decoot 0,837. Grm., 100,0 Lieber 1,58 Grm., di e ganze Leber 1,98 Grm. Zucker.

II. Stück, $73,0 \mathrm{Grm}$, wird $1 \frac{1 / 2}{2}$ Stunden später verarbeitet. Es liefert 1580,0 C.-Ctm. Decoct. Davon reduciren nach dem. Enteiweissen 26,0 C.-Ctm. 5,0 C.-Ctm. Fehling'scher Lösung. Demnach enthalt das Decoet 1,517 Grm., 100,0 Lober 2,08 Grm., die ganze Leber 2,61 Grm. Zucker.

Der Zuckergehalt der Leber hat sonach innerhalb $1 \frac{1 / 2}{2}$ Stunden von 1,58 pOt. bis 2,08 pOt., also um 0,5 pCt. zugenom men.

Ergibt sich aus diesen beiden Versuchen in voller Uebereinstimmung mit den Angaben Schiff's und Bernard's eine rasche Zunahme des Leberzuckergehaltes nach dem Tode, so zeigt der nachfolgende Versuch, dass diese Vermebrung auf Kosten des Glykogengehaltes der Leber stattfindet. Zugleich kann man aus dem Resultat dieses Versuches entnehmen, dass wenigstens in den ersten 3 Stunden nach dem Tode kein e erhebliche Zersetzung des gebildeten Zuckers Platz greift. Die Versuchsanordnung war die gleiche, wie in den beiden vorausgehenden Versuchen, nur wurde hier aus beiden Leberstïcken auch das Glykogen nach der Briucke'schen Methode dargestellt und quantitativ bestimmt.

Versueh CXXXIII. 13/I. 77. Katze von 2,60 Kilo, mit Rindfleisch gefüttert. Leber $154,0 \mathrm{Grm}$. schwer $=1 / 17$ des Rörpergewichtes.

I. Stück der Leber 73,0 Grm. schwer gibt 3040,0 O.-Ctm. Decoct. 100,0 C.-Ctm. davon enteiweisst geben nach dem Enteiweissen 70,0 C.-Ctm. Davon reduciren 27,0 C.-Ctm. 2,0 C.-Ctm. Fehling'scher Lösung. Das 
I. Leberstïck enthält demnach $0,7873 \mathrm{Grm}$. 100,0 Leber 1,07 Grm., die ganze Leber $1,65 \mathrm{Grm}$. Zu cker.

Ans 2940,0 C.-Ctm. erhält man 7,761 Grm. Glykogen, 3040,0 C.-Ctm. enthalten daher 8,02 Grm., die ganze Leber 16,91 Grm. oder 10,98 pCt. Glykogen.

II. Stück der Leber $81,0 \mathrm{Grm}$. schwer, 3 Stunden später in Arbeit genommen, liefert 3340,0 C.-Ctm. Decoct; nach dem Enteiweissen reduciren 19,4 C.-Ctm. 2,0 C.-Ctm. Fehling'scher Lösung. Das II. Lebersttick enthält daher 1,70 Grm., 100,0 Leber 2,0, die ganze Leber 3,2 Grm. Zneker.

Aus 3240,0 C.-Ctm. Decoct erhält man 8,143 Grm. Glykogen, entsprechend einem Glykogengehalt des II. Leborstî̀ cks von $8,39 \mathrm{Gr}$., und der ganzen Leber von $15,95 \mathrm{Grm}$. oder $10,03 \mathrm{pCt}$.

Der Zuckergehalt der Leber hat sonach innerhalb 3 Stunden von $1,07-2,0$ pCt., also $\mathrm{nm} 0,93 \mathrm{pCt}$. zu genommen, der Glykogengehalt hingegen ist von $10,98 \mathrm{pCt}$. auf $10,03 \mathrm{pCt}$. also um 0,95 p Ct. gesunken.

Berechnen wir aus obigen Daten a) den Gesammtkohlehydratbestand der ganzen Leber, indem wir Glykogen auf Zucker umrechnen aus den Resultaten für das I. Leberstiuck: 1. Zncker 1,65 Grm.

2. Glykogen 16,91 als Zucker . 18,87 so ergibt sich die Gesammtsumme von . . 20,52 "

b) aus den Resnltaten für das II. Leberstitck berechnet:

1. Zucker . . . . . . . . . 3,2 Grm.

2. 15,95 Grm. Glykogen als Zucker 17,70 "

so ergibt sich die Summe vol . • . 20,90"

Es wurden also nach der Bestimmung des II. Leberstücks $0,48 \mathrm{Grm}$. Zucker mehr gefunden, ein Fehler, der wahrscheinlich auf einen kleinen Glykogenverlust bei der Verarbeitung des ersten Leberstücks zuritekzuftihren ist. Beide Bestimmungen stimmen himreichend genau überein um zu zeigen, dass in der That der Zunahme des Zuckergehaltes auf Kosten des Glykogengehaltes der Lebor stattgefunden hat.

Aus den in Tabelle III zusammengestellten 29 Leberzuckerbestimmungen berechnet sich das durchschnittliche Verhältuiss des Leberzucker- zum Körpergewicht auf 0,53. Aus 18 Beobachtungen, wo uns auch das Gewicht der frischen Leber bekannt war, erhalten wir als durchschnittlichen Zuckergehalt der frischen Leber 1,42 pCt. ') Aus dem oben Gesagten g'eht tubrigens hervor, dass diese Durchschmittszahl nur einen geringen Werth beanspruchen kann, und dass die auffallende Uebereinstimmung zahlreicher Leberzuckerbestimmungen nicht etwa in einem annäher'nd gleichen Zuckergehalt der Leber, sondern darin ihren Grund hat, dass in den meisten Lebern annä-

1) Dieser Zahl kommt eine ältere Angabe Cl. Bernard's nahe genug; er fand im Durchschnitt $1,5-2,0 \mathrm{pCt}$. bei seinen zahlreichen Bestimmungen an den verschiedensten Süugethieren und Vögeln. Leçons de physiologie expérimentale. Vol. I. p. 64 . 
herud eine gleiche Menge von Glykogen in Zucker übergegangen war. Ueberraschend istes dabei zu sehen, dass die Zuckermenge, welche man in einer Leber findet, nicht direct von der vorhandenen Glykogenmenge abhängt. Eine sebr glykogenreiche Leber liefert nicht mehr Zucker als eine glykogenarme. So fanden sich, um einige Beispiele anzufuhren, in Versuch 76 neben 9,41 Grm. Glykogen 1,74 Grm. Zucker, in Versuch 130 neben 21,7 Grm. Glykogen 2,48 Grm. Zucker, in Versuch 134 endlich neben 1,8 Grm. Glykogen 1,73 Grm. Zucker. Aus sehr glykogenarmen Lebern schwindet sogar häufig das Glykogen vollständig auf Kosten des gebildeten Zuckers. Es finden sich aber unter unseren Versuchen zahlreiche Falle wo die völlig glykogenfreie Leber $1,0-1,5 \mathrm{Grm}$. Zucker enthielt. Daraus erklärt sich auch die auffallende Constanz des Verhältnisses der in der Leber vorgefundenen Zuckermengen zum Körpergewicht, welche nicht möglich wäre, wenn eine glykogenarme Leber relativ weniger Zucker producirte als eine glykogenreiche.

Die Langwierigkeit und Kostspieligkeit der Glykogenbestimmungen brachte es mit sich, dass wir solche nur in relativ beschränkter Anzahl ausfuhren konnten. Tabelle IV gibt die Zusammenstellung von 18 Glykogenbestimmungen normaler Thiere.

Tabelle IV.

\begin{tabular}{|c|c|c|c|c|c|}
\hline 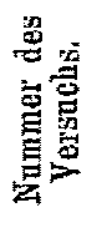 & $\begin{array}{l}\text { koppergowicht } \\
\text { in Kilogramm. }\end{array}$ & $\begin{array}{l}\text { Lebergewicht } \\
\text { in Grm. }\end{array}$ & $\begin{array}{c}\text { Glykogengehalt } \\
\text { der } \\
\text { Lober } \\
\text { in Gru. }\end{array}$ & $\begin{array}{c}\text { Glykogengelialt } \\
\text { in pCt. } \\
\text { des Gewiohts } \\
\text { der } \\
\text { frischen Eeber. }\end{array}$ & $\begin{array}{l}\text { Körpor- } \\
\text { gewicht } \\
\text { dividirt durch } \\
\text { Lebergervicht. }\end{array}$ \\
\hline $\begin{array}{r}133 \\
130 \\
66 \\
84 \\
76 \\
118 \\
89 \\
69 \\
77 \\
87 \\
142 \\
85 \\
135 \\
78 \\
141 \\
144 \\
134 \\
71\end{array}$ & $\begin{array}{l}2,60 \\
4,07 \\
2,89 \\
2,29 \\
3,12 \\
2,2 \\
2,1 \\
3,6 \\
3,02 \\
2,07 \\
2,55 \\
2,72 \\
2,38 \\
2,72 \\
3,83 \\
2,75 \\
2,79 \\
2,27\end{array}$ & $\begin{array}{r}154 \\
205 \\
148,6 \\
100,0 \\
127,1 \\
83,7 \\
80,3 \\
139,5 \\
112,1 \\
72,0 \\
83,0 \\
90,5 \\
75,0 \\
87,1 \\
114,0 \\
82,0 \\
80,4 \\
59,3\end{array}$ & $\begin{array}{c}16,91 \\
21,70 \\
7,92 \\
2,73 \\
9,41 \\
3,9 \\
1,35 \\
2,9 \\
1,67 \\
1,0 \\
2,13 \\
2,71 \\
3,64 \\
1,85 \\
1,91 \\
3,52 \\
1,8 \\
1,69\end{array}$ & $\begin{array}{r}10,9 \\
10,5 \\
5,3 \\
2,7 \\
7,4 \\
4,7 \\
1,6 \\
2,1 \\
1,4 \\
1,4 \\
2,5 \\
3,0 \\
4,8 \\
2,1 \\
1,5 \\
4,2 \\
2,2 \\
2,8\end{array}$ & $\begin{array}{l}1 / 16 \\
1 / 10 \\
1 / 19 \\
1 / 29 \\
1 / 27 \\
1 / 26 \\
1 / 26 \\
1 / 26 \\
1 / 24 \\
1 / 28 \\
1 / 28 \\
1 / 30 \\
1 / 31 \\
1 / 33 \\
1 / 33 \\
1 / 33 \\
1 / 34 \\
1 / 38\end{array}$ \\
\hline
\end{tabular}

Man ersieht aus dieser Tabelle auf den ersten Blick die enormen Schwankungen, denen der Glykogengehalt der Leber unterworfen ist 
(1,4-10,9 pCt. der frischen Leber). Es lässt sich also auch trotz sorgfultigster gleichmässiger Ernährung in dieser Beziehung keine annähernde Constanz erzielen.

Sehr glylkogenreiche Lebern zeìchnen sich zunächst schon durch ihr relativ hohes Gewicht aus. In Tabelle $\mathrm{V}$ haben wir alle Zahlen zusammengestellt, welche einerseits über das Verhältniss des Körpergewichts zum Lebergewicht, andererseits uber die Beziehung des Lebergewichts zum Kohlehydratbestand der Lieber Aufschluss geben. Die Versuche sind nach dem Lebergewicht in ansteigender Reihe geordnet.

Tabelle V.

\begin{tabular}{|c|c|c|c|}
\hline $\begin{array}{l}\text { Nummer des } \\
\text { Versuchs. }\end{array}$ & $\begin{array}{l}\text { Gewicht } \\
\text { der. } \\
\text { Leber } \\
\text { in Grm. }\end{array}$ & $\begin{array}{l}\text { Köorpergewiclit } \\
\text { dividirt } \\
\text { durch das } \\
\text { Leberg ewicht. }\end{array}$ & $\begin{array}{l}\text { Kohlehydratbestand } \\
\text { der Lieber } \\
\text { in pCt. des Gewichts } \\
\text { der frischen Leber. }\end{array}$ \\
\hline $\begin{array}{r}133 \\
130 \\
66 \\
131 \\
84 \\
132 \\
76 \\
55 \\
80 \\
67 \\
143 \\
89 \\
69 \\
86 \\
77 \\
87 \\
75 \\
126 \\
127 \\
56 \\
81 \\
117 \\
142 \\
85 \\
123 \\
135 \\
109 \\
65 \\
49 \\
113 \\
106 \\
141 \\
144 \\
79 \\
88 \\
121 \\
134 \\
114\end{array}$ & $\begin{array}{r}154,0 \\
205,0 \\
148,0 \\
116,0 \\
100,0 \\
120,0 \\
127,0 \\
128,0 \\
96,0 \\
113,3 \\
82,7 \\
80,3 \\
139,5 \\
100,0 \\
112,1 \\
72,0 \\
90,0 \\
90,5 \\
102,0 \\
118,0 \\
81,0 \\
84,0 \\
83,0 \\
90,5 \\
86,0 \\
75,0 \\
84,0 \\
87,1 \\
42,0 \\
81,0 \\
76,0 \\
114,0 \\
82,0 \\
109,5 \\
70,5 \\
74,0 \\
80,4 \\
83,0\end{array}$ & $\begin{array}{l}1 / 16 \\
1 / 10 \\
1 / 10 \\
1 / 24 \\
1 / 21 \\
1 / 23 \\
1 / 24 \\
1 / 25 \\
1 / 25 \\
1 / 26 \\
1 / 26 \\
1 / 26 \\
1 / 20 \\
1 / 27 \\
1 / 27 \\
1 / 28 \\
1 / 28 \\
1 / 29 \\
1 / 28 \\
1 / 20 \\
1 / 30 \\
1 / 30 \\
1 / 30 \\
1 / 30 \\
1 / 30 \\
1 / 31 \\
1 / 31 \\
1 / 32 \\
1 / 32 \\
1 / 39 \\
1 / 33 \\
1 / 33 \\
1 / 33 \\
1 / 33 \\
1 / 33 \\
1 / 33 \\
1 / 34 \\
1 / 34\end{array}$ & 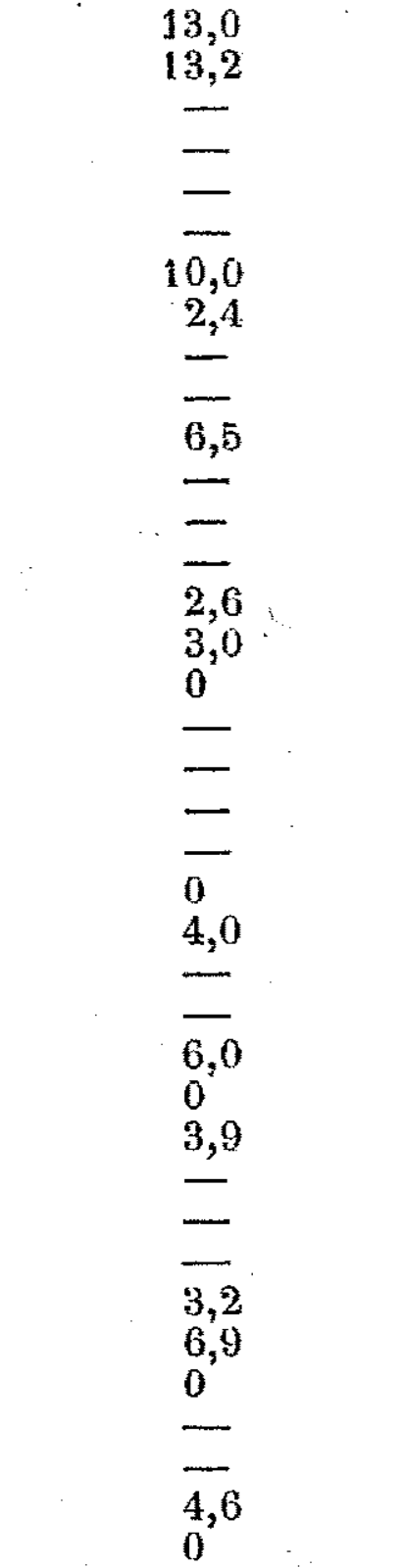 \\
\hline
\end{tabular}


Beiträge zur Kenntniss des Kohlehydratstoffwechsels.

\begin{tabular}{|c|c|c|c|}
\hline $\begin{array}{l}\text { Nummer des } \\
\text { Versuchs. }\end{array}$ & $\begin{array}{l}\text { Gewicht } \\
\text { der } \\
\text { Lober } \\
\text { in Grm. }\end{array}$ & $\begin{array}{l}\text { Körpergewicht } \\
\text { dividirt } \\
\text { durch das } \\
\text { Lebergewicht. }\end{array}$ & $\begin{array}{l}\text { Kohlehyd ratbestand } \\
\text { der Leber } \\
\text { is pCt. des Gewichts } \\
\text { der frischen Leber. }\end{array}$ \\
\hline $\begin{array}{r}89 \\
99 \\
106 \\
129 \\
108 \\
112 \\
105 \\
120 \\
74 \\
70 \\
71 \\
140 \\
115 \\
110 \\
107 \\
104 \\
128 \\
102 \\
92 \\
72 \\
103\end{array}$ & $\begin{array}{r}64,0 \\
87,2 \\
76,0 \\
82,0 \\
78,0 \\
72,0 \\
75,0 \\
62,0 \\
87,5 \\
44,0 \\
59,5 \\
107,0 \\
60,0 \\
63,0 \\
73,0 \\
73,0 \\
89,0 \\
96,5 \\
55,0 \\
52,0 \\
48,0\end{array}$ & $\begin{array}{l}1 / 34 \\
1 / 34 \\
1 / 34 \\
1 / 34 \\
1 / 36 \\
1 / 36 \\
1 / 30 \\
1 / 37 \\
1 / 37 \\
1 / 37 \\
1 / 39 \\
1 / 38 \\
1 / 39 \\
1 / 41 \\
1 / 41 \\
1 / 42 \\
1 / 44 \\
1 / 46 \\
1 / 433 \\
1 / 50 \\
1 / 54\end{array}$ & $\begin{array}{l}- \\
\bar{Z} \\
\bar{Z} \\
\overline{0} \\
0 \\
0 \\
0 \\
- \\
1,2 \\
0,1 \\
2,1 \\
0 \\
0 \\
- \\
0 \\
-\end{array}$ \\
\hline
\end{tabular}

Man bemerkt zunächst, dass schon das Lebergewicht an sich sehr bedeutend variirt, und zwav zwischen $1 / 16-1 / 54$ des Körpergewichtes. ${ }^{1}$ ) Sodann sehen wir, dass in der Regel sehr hohes Lebergewicht mit einem hohen Kohlehydratgehalt einhergeht. Die schwersten Lebern, die wir bei unseren Versuchen fanden (Versuch 133 1/10 des Körpergewichts, und Versuch 130 1/10 des Körpergewichts) enthielten auch unter allen Versuchen die grösste Menge von Kohlehydraten (13,0 und 13,2 pCt.). Indessen findet sich, dass erst dann die Kohlehydratmenge eine sehr geringe wird, wemn das Lebergewicht unter $1 / 30$ des Körpergewichts herabsinkt, obwohl wir auch Lebern begegneten, welche bei relativ hohem Gewicht ganz frei von Kohlehydraten gefunden wuxden (vgl. Versuch $75,117,83,109,79$ ) - allerdings lauter Versuche, wo das Verschwinden der Kohlehydrate aus der Leber durch experimentelle Eingriffe verursacht war.

Glykogenreiche Lebern sind ausserdem in der Regel voluminöser,

1) Das Gewicht der menschlichen Leber schwankt zwischen 1/2,-1/40 des Körpergewichts und scheint nach Frerichs (Klinil der Leberkrankh. I. S. 18, 32) im nüchternen Zustande geringer zu sein als nach Aufnahme von Nahrung. - Nasse (Arch. d. Vereins f. gemeinsch. Arbeiten IV. 79) verglich das Gewicht der Leber bei gefütterten und hungerndën Kaninchen; es betrug im Mittel dort 43,53 , hier nur 35,12 pro Mille des Körpergewichts. - Henle, Handbuch der systemat. Anatomie. Bd. II. S. 191. 
weicher und heller gefärbt als glykogenarme. Dass der Glykogengehalt der Leber von dem Ernährungsstand der Thiere sehr abhängig ist, ist genugsam belannt. Hunger und pathologische Vorgänge vermögen ihn rasch zu reduciren oder ganz zum Verschwinden zu bringen.

Eine bisher noch nicht beachtete Beziehung des Glykog'engehaltes der Leber zum Ernährungsstand ist uns in zahlreichen Versuchen in sehr auffälliger Weise entgegengetreten. Wenn man schlecht genährte, magere oder längere Zeit vorher hungernde Katzen mehrere Tage hindurch sorgfältig mit frischem Fleisch fiittert, so nimmt zumeist ihr Körpergewicht rasch zu und man constatirt alsbald grosse Kohlehydratrorratthe in der Leber. Als Beispiel mag folgender Versuch dienen (No. 76). Eine kräftige Katze, seit mehreren Wochen im Käfig mit Fleisch gefïttert, wog am 7. April 1876 2,95 Kilo. Vom 7.-11. April hungerte das Thier, und das Körpergewicht sank in Folge dessen anf 2,5 Kilo. Vom 11. April an bekam es täglich wieder reichlich Pferdefleisch und wog am Tage des Versuches, den 19. April 1876 wieder 3,12 Kilo. Man fand in der Leber 9,41 Grm. Glykogen und 1,8 Grm. Zucker (10,0 pCt. Kohlehydrate). - Wird nun aber die gleiche Fitttermg fortgesetzt, bis das Thier reichlich Fett ansetzt (was naturlich je nach der Individualität des Thieres verschieden leicht eintritt, zuweilen auch ganz ausbleibt), so findet man im weiteren Verlanfe diẻ Leber um so glykogenärmer, je reichlicher die Fettablagerung stattgefunden hat. Hierfür liefert Versuch No. 140 ein schlagendes Beispiel. Das Thier, eine ungemein kräftige Katze von 2,79 Kilo, befand sich schon seit zwei Mouaten in der Fleischfitterung, als es am 17./I. 77. zum Versuch benitzt wurde. Im Netz sowie im übrigen Körper fanden sich enorme'Fettmassen abgelagert, die Leber enthielt aber nur 1,8 Grm. Glykogen. Noch auffallender war dieses Verhalten im Versuch No. 140. Er betraf einen 4,07 Kilo schweren, enorm fetten Kater der keine Spur von Glykogen in seiner Leber besass.

Das Verhältniss ist ein so constantes, dass man sicher sein kann, bei sehr fetten Thieren niemals eine grössere Menge von Leberglykogen zu finden. Es erscheint uns nicht tiberflitssig, hinzuzufügen, dass unsere Versuche fast ohne Ausnahme an nüchternen Katzen angestellt wurden. Unmittelbar oder wenige Stunden nach der Fiutterung, also während der Verdauung, welche nach Cl. B ernard immer eine Vermehrung des Leberglykogens zur Folge hat, wird man wahrscheinlich auch bei fetten Thieren grössere Glykogenmengen vorfinden. 
Hieraus wiirde sich nun, auch bei ausschliesslicher: Fleischnahrung, die Möglichkeit einer doppelten Art der Aufspeicherung überschtissig' zugefthrter Nahrungsstoffe in Form stickstofffreier Substanzen ergeben. Die Leber wiurde aber bei einem Thiere nur so lange die Rolle einer bedeutsameren Vorrathskammer spielen, bis eine nene und wahrscheinlich beständigere Anlage des iberschiissigen Materials in For'm von Fettgewebe stattfindet.

Den in Tabelle VI zusammengestellten Muskelglykogenbestimmungen haben wir nichts Wesentliches binzuzuftigen. Sie zeigen, wie die analogen Befunde von $\mathrm{W}$ eiss ${ }^{1}$ ) und $\mathrm{Nasse}{ }^{2}$ ), dass auch an eine annähernde Constanz des Muskelglykogenbestandes nicht gedacht werden kann, dass tibrigens doch das in dieser Form im Körper deponirte stickstofffreie Material hänfig sehr erhebliche Werthe repräisentirt.

Tabelle VI.

\begin{tabular}{|c|c|c|c|c|c|}
\hline 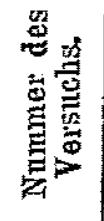 & $\begin{array}{c}\text { Gewicht } \\
\text { des } \\
\text { Thieres } \\
\text { in Kilogramon. }\end{array}$ & $\begin{array}{c}\text { Dargestelltes } \\
\text { Glylrogen } \\
\text { in Grm. }\end{array}$ & $\begin{array}{l}\text { Gewiclit } \\
\text { der } \\
\text { Gesammt- } \\
\text { museulatur } \\
\text { in Grm. }\end{array}$ & $\begin{array}{c}\text { Gehalt } \\
\text { aller MInskeln } \\
\text { an Glykogen: } \\
\text { in Grm. }\end{array}$ & $\begin{array}{l}\text { Glykogengelialt } \\
\text { des } \\
\text { Aruskelgowelies } \\
\text { in pCt. }\end{array}$ \\
\hline $\begin{array}{r}68 \\
76 \\
87 \\
130 \\
134 \\
135\end{array}$ & $\begin{array}{l}2,72 \\
3,12 \\
2,07 \\
4,07 \\
2,79 \\
2,38\end{array}$ & $\begin{array}{l}0,193 \\
0,536 \\
0,426 \\
1,52 \\
0,431 \\
0,80\end{array}$ & $\begin{array}{c}1090,0 \\
1204,0^{*} \\
828,0 \\
1628,0 \\
1130,0^{*} \\
908,9\end{array}$ & $\begin{array}{l}3,2 \\
3,27 \\
0,93 \\
6,7 \\
1,6 \\
2,85\end{array}$ & $\begin{array}{l}0,29 \\
0,27 \\
0,11 \\
0,41 \\
0,14 \\
0,30\end{array}$ \\
\hline
\end{tabular}

*) Die mit einem * bezeichneten Zahlen sind durch sorgfältiges Abpräpariren der Gesammtmusculatur erhalten, die übrigen berechnet (Muskelgewicht $=$ 0,4 des Ixörpergervichts).

Diejenigen Versuche endlich, welche tiber den Gesammtkohlenhydratbestand des Katzenkörpers Aufschluss geeben, sind in Tabelle VII zusammengestellt. Man ersieht daraus, dass die Menge der im Tabelle VII.

\begin{tabular}{|c|c|c|c|c|c|c|c|c|c|c|}
\hline \multirow{2}{*}{ 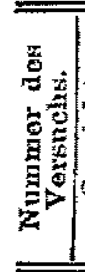 } & \multirow{2}{*}{ 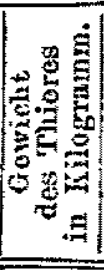 } & \multirow{2}{*}{$\begin{array}{c}: \\
\text { Art } \\
\text { der } \\
\text { Futterung. }\end{array}$} & \multicolumn{2}{|c|}{ Zucker im Blut: } & \multicolumn{2}{|c|}{ Leber: } & \multirow{2}{*}{ 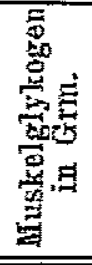 } & \multirow{2}{*}{ 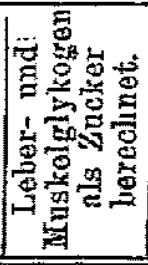 } & \multicolumn{2}{|c|}{$\begin{array}{l}\text { Gesammtkohlehydrato } \\
\text { in Grm. Zucker } \\
\text { ausgedruckt. }\end{array}$} \\
\hline & & & pCt. & $\begin{array}{l}\text { im } \\
\text { Gesammt- } \\
\text { blut } \\
\text { in Grm. }\end{array}$ & $\begin{array}{l}\text { Zuckor } \\
\text { in Grm. }\end{array}$ & $\begin{array}{l}\text { Glykogen } \\
\text { in Grm. }\end{array}$ & & & $\begin{array}{l}\text { Im Thiere } \\
\text { vorge- } \\
\text { funden. }\end{array}$ & $\begin{array}{l}\text { pro I Kilo } \\
\text { Iintze } \\
\text { berechnot. }\end{array}$ \\
\hline $\begin{array}{r}76 \\
65 \\
87 \\
130 \\
134 \\
135\end{array}$ & $\left\{\begin{array}{l}3,12 \\
2,72 \\
2,07 \\
4,07 \\
2,79 \\
2,38\end{array}\right.$ & $\begin{array}{l}\text { Pferdefleisch } \\
\text { do. } \\
\text { Rindfleisch. } \\
\text { do. } \\
\text { do. } \\
\text { do. }\end{array}$ & $\begin{array}{l}0,13 \\
0,13 \\
0,13 \\
0,26 \\
0,21 \\
0,21\end{array}$ & $\begin{array}{l}0,33 \\
0,27 \\
0,20 \\
0,81 \\
0,44 \\
0,38\end{array}$ & $\begin{array}{l}1,74 \\
1,28 \\
1,03 \\
2,48 \\
1,73 \\
1,36\end{array}$ & $\begin{array}{c}9,41 \\
1,85 \\
1,0 \\
21,7 \\
1,8 \\
3,64\end{array}$ & $\begin{array}{l}3,27 \\
3,2 \\
0,93 \\
6,7 \\
1,6 \\
2,85\end{array}$ & $\begin{array}{r}14,08 \\
5,61 \\
2,14 \\
31,52 \\
3,77 \\
7,20\end{array}$ & $\begin{array}{r}16,16 \\
7,16 \\
3,40 \\
34,80 \\
5,95 \\
8,94\end{array}$ & $\begin{array}{l}5,1 \\
2,6 \\
1,6 \\
8,5 \\
2,1 \\
3,7\end{array}$ \\
\hline
\end{tabular}

1) Wiener Sitzungsber., math.-phys. Cl. Tom. LXIV. 1871.

2) Pfliger's Arch. Bd. VII. 
Körper in einem gegebenen Moment ausserhalb der Verdauung vorhandenen Kohlehydrate $1,5-8,5$ Grm. pro Kilo Katze betragen kann.

\section{ANHANG.}

Protokolle und analytische Belege.

I. Gruppe. Versuche, in welchen der Gesammtkohlehydratbestand des Körpers ermittelt wurde (vgl. Tabelle VII).

1. Fersuch (No. LXVIII). 30/III. 76. Kater von 2,72 Kilo. Pferdefleischfitterung.

A. 35,59 Grm. Blut aus der Carotis geben 185,0 C.-Ctm. Decoct. 19,0 C.-Ctm. reduciren 1,0 C.-Ctm. F. L. ${ }^{1}$ ) also 35,59 Blut $=$ 0,048 Grm. Zucker $=0,13$ pOt. Gesammtblut $=207,0$ Grm. 0,27 Grm. Z. ${ }^{2}$ )

B. Leber (87,1 Grm.) gibt $13.05,0$ Decoct. a) 625,5 Decoct, mit Alkohol enteiweisst, geben 400,0 C.-Ctm.; davon reduciren 9,3 O.-Ctm. 3,0 C.-Ctm. F. L. also 625,5 C.-Ctm. Decoct $=0,644$ Grm. Z. Die ganze Leber 1,288 Grm. Z. (1,4 pCt.). b) 625,5 Decoct liefern $0,929 \mathrm{Gr}$. Glykogen also die ganze Leber $1,859(=2,063 \mathrm{Grm} . \mathrm{Z}$ )

C. Muskeln 128,5 Grm. geben 0,386 Grm. Glykogen. Gesammtmuskeln $(0,4$ des Körpergewichts $=1090,0$ Grm. $)=3,2 \mathrm{Grm}$. Glykogen (=3,55 Grm. Z.).

$$
\begin{aligned}
& \text { A. Blut }=0,27 \\
& \text { B. a) (Leber) }=1,28 \\
& \text { b) }=2,06 \\
& \text { C. Muskel }=3,55 \\
& \hline
\end{aligned}
$$

2. Versuch (No. LXXVI). 19/IV. 76. Kater von 3,12 Kilo. Pferdefleischfütterung.

A. 43,17 Grm. Blut aus der Carotis geben 300,0 C.-Ctm. Decoct. 25,0 C.-Ctm. reduciren 1,0 C.-Ctm. F. L. also 43,17 Grm. Blut $=0,06$ Grm. Z. $=0,13$ pCt. Gesammtblut 240,0 Grm. $=0,331$ Grm. Z.

B. Leber (127,1 Grm.) gibt 1960,0 C.-Ctm. Decoct. a) 300 C.-Ctm. Decoct nach dem Enteiweissen durch Alkohol 315,0 O.-Ctm. 17,0 C.-Ctm. reduciren 3,0 C.-Ctm. F. L. Also 300,0 Decoct $=0,267$ Grm. Z. 1960,0 Decoct (ganze Leber) $=1,74$ Grm. Z. $=1,3$ pCt. b) 1500,0 C.-Ctm. Decoct liefern 8,597 Grm. Glykogen; also die ganze Leber 9,419 Grm. (= 10,455 Grm. Z.).

C. Muskeln 207,0 Grm. liefern 0,563 Grm. Glykogen. GesammtmuskeIn 1204,0 Grm. $=3,274$ Grm. Glylkogen $=3,634$ Grm. Z.)
A. Blut
B. Leber 0,331
a) 1,740
b) 10,455
C. Muskeln 3,634
16,160 oder 5,1 pro 1 Kilo Katze.
1) F. L. = Fehling'sche Lösung.
2) Z. = Zucker. 
3. Versuch (No, LXXXVII). Katze von 2,07 Kilo. Rindfleischfutterung.

A. Die Bestimmung des Blutzuckers missgltickt.

B. L eber (72,0 Grm.) gibt 1400,0 C.-Ctm. Decoct. a) zwei Portionen von je 100,0 C.-Ctm. Decoct mit zwei verschiedenen Sorten Thierkohle behandelt ( $\alpha$ und $\beta$ ). $\alpha$ gibt 445,0 C.-Ctm. Decoct. 30,0 C.-Ctm. reduciren 1,0 C.-Ctm. F. L. Demnach 100,0 Leberdecoct $=0,074$ Grm. Z., die ganze Leber 1,0 Grm. Z. - $\beta$ gibt 320,0 Decoct, wovon 22,0 C.-Ctm. 1,0 C.-Ctm. F. L. reduciren; also hiernach 100,0 Leberdecoct $=0,073 \mathrm{Grm}$. Z., die ganze Leber 1,02 Grm. Z. 100 C.-Ctm. Decoct werden direct titrirt. 6,5 C.-Ctm. reduciren 1,0 C.-Ctm. F. L., demnach also 100,0 Decoct $0,076 \mathrm{Grm}$. Z., die ganze Leber 1,06 Grm. Z. b) 1100,0 0.-Ctm. Decoct geben $0,715 \mathrm{Grm}$. Glykogen, die ganze Leber 1,0 Grm. $(=1,11 \mathrm{Grm} . \mathrm{Z}$.$) .$

C. Muskeln 376,5 Grm. geben 0,426 Grm. Glykogen, Gesammtmuskeln ( $=0,4$ des Körpergewichts oder 828,0 Grm.) 0,93 Grm. Glykogen (=1,03 Grm. Zi).

Zur Completirung des Versuchs wird der Zuckergehalt des Blutes auf 0,13 pCt., Gesammtblut 0,20 Grm. Z. angenommen.
A. Blut
0,20
B. Leber a)
1,06
b) 1,11
C. Muskeln
1,03
3,40 oder 1,6 pro 1 Kilo Katze.

4. Versuch (No. CXXX). Kater von 4,07 Kilo. Rindfleischfttterung. A. 26,12 Grm. Blut ans der Carotis, geben 155,0 O.-Ctm. Decoct. 11,3 C.-Ctm. reduciren 1,0 C.-Ctm. F. L. also 26,12 Grm. Blut $=0,069$ Grm. $=0,26$ pCt. Z. Gesammtblnt (313,0 Grm.) $=$ 0,813 Grm. Z.

B. Leber $(205,0$ Grm.) gibt 2085,0 C.-Ctm. Decoct. a) von 105,0 C.-Ctm. Decoct, nach dem Enteiweissen durch Alkohol 100,0 reduciren 20,0 C.-Ctm. 5,0 C.-Ctm. F. L. 105 C.-Ctm. Decoct also $=0,125$ Grm. Ganze Leber (2085,0 Decoct) 2,48 Grm. Zucker $(1,2$ pCt.). b) 1980,0 C.-Ctm. Decoct geben 20,682 Grm. Glykogen, die ganze Leber 21,7 Grm. Glykogen (-24,08 Grm. Z.).

C. Muskeln $(367,0$ Grm.) liefern 1,520 Grm., die ganze Musculatur 1628,0 Grm. 6,7 Grm. Glylkogen $(=7,43$ Grm. Z.).
A. Blut
$=0,813$
B. Leber a) $=2,480$
b) $=24,080$
C. Muskeln $=\frac{7,430}{34,803}$
$=8,5$ pro 1 Kilo Katze.

5. Versuch (No. CXXXIV). Katze von 2,79 Kilo. Rindfleischfiitterung. A. 25,58 Gr.m. Blut aus der Carotis geben 215,0 C.-Ctm. Decoct; 20,0 C.-Ctm. reduciren 1,0. C.-Ctm. F. L. also 25,58 Grm. Blut $=0,053 \mathrm{Grm} .=0,21$ pCt. Z. Gesammtblut 214,0 Grm. $=$ $0,449 \mathrm{Gl}$. $\mathrm{Z}$.

B. Leber $(80,4$ Grm. $)$ gibt 2440,0 C.-Ctm. Decoct; a) von 100,0 C.-Ctm. Decoct reduciren nach dem Enteiweissen u. s. w. durch: 
Alkohol 21,0 C.-Ctm. 3,0 C.-Ctm. F. L. Die Leber enthält demnach 1,732 Grm. Z. $=2,1$ pCt. b) 2340,0 C.-Ctm. Decoct liefern 1,726 Grm. Glykogen, die ganze Leber also 1,8 Grm. = 1,99 Grm. Z.)

C. Muskeln 290,4 Grm. liefern 0,431 Grm. Glykogen. Gesammtmusculatur $1130,0 \mathrm{Grm}$. (bestimmt) 1,6 Grm. ( $=1,77$ Z.).

$$
\begin{array}{ll}
\text { A. Blut } & =0,449 \\
\text { B. Leber a) } & =1,732 \\
\text { C. Muskeln } & =1,998 \\
& =1,77 \\
5,949 \mathrm{Grm} . \mathrm{Z}_{*}=\operatorname{oder} 2,1 \text { pro } 1 \text { Kilo Katzo. }
\end{array}
$$

6. Versuch (No. CXXXV). 19/I. 77. Katze von 2,38 Kilo. Rindfleischfütterung.

A. 25,38 Grm. Blut geben 205,0 Decoct; 19,0 C.-Ctm. reduciren 1,0 C.-Ctm. F. L. 25,38 Blut demnach $=0,026$ Grm. $=0,21$ pCt. Z. Gesammtblut $=183$ Grm. $=0,384$ Grm. Z.

B. Leber $(75,0$ Grm.) gibt 400,0 Decoct. a) von 100,0 C.-Ctm. Decoct reduciren nach dem Enteiweissen u. s. w. 14,5 C.-Ctm. F. L., die Leber enthält demnach 1,36 Grm. Z. (=1,8 pCt.), b) 3900 C.-Ctm. Decoct geben 3,56 Grm. Glykogen, die ganze Leber demriach 3,64 Grm. ( 4,04 Grm. Z.).

C. Muskeln 393,0 Grm. geben 0,802 Grm. Glykogen, Gesammtmusculatur 908,0 Grm. (bestimmt) 2,85 Grm. (=.3,16 Grm. Z.).

$$
\begin{array}{ll}
\text { A. Blut } & 0,384 \\
\text { B. Leber a) } & 1,36 \\
\text { C. Muskeln } & 4,04 \\
\frac{8,16}{8,94 \mathrm{Gr}} & \text { Z. oder } 3,7 \text { pro } 1 \text { Kilo Katze. }
\end{array}
$$

II. Gruppe. Versuche, in welchen nur der Zuckergehalt des Blutes und der Leber ermittelt wurde. fleischfütterung.

1. Versuch (No. LII). 5/III. 76. Katze von 2,76 Kilo. Pferde-

A. B1 $u$ t enthalt $0,13 \mathrm{pCt}$. Z. Gesammtblut $=212,0 \mathrm{Grm} .0,265 \mathrm{Grm} . \mathrm{Z}$.

B. Leber liefert 550,0 C.-Ctm. Decoct mit 1,42 Grm. Z. Glykogen fehlt.

$$
\begin{array}{ll}
\text { A. Blut } & 0,26 \\
\text { B. Leber } & 1,42 \\
\hline & 1,68(0,68 \text { pro Kilo. }
\end{array}
$$

2. Versuch (No. LIII). 5/MI. 76. Katze von 1,65 Kilo. Früher Pferdefleischfitterung

$$
\text { seit } 3 \text { Tagen Hunger. }
$$

A. Blut enthält 0,18 pCt. Z. Gesammtblut $=126,0$ Grm. $=0,23$ Grm. Z.

B. Leber enthalt 0,76 Grm. Z.

$$
\begin{array}{ll}
\text { A. Blut } & 0,23 \\
\text { B. Leber } 0,76
\end{array}
$$

0,99 Grm. Z. oder 0,61 pro 1 Kilo Katze.

3. Versuch (No. LIV). 8/III. 76. Früher Pferdefleischfütterung; seit 3 Tagen Hunger. 
A. B I it enthält 0,13 pCt. Z. Gesammtblut $=139,0 \mathrm{Grm} .=0,167 \mathrm{Grm} . \mathrm{Z}$.

B. Leber enthält 0,39 Grm. Zucker, kein Glykogen.
A. Blut 0,167
B. Leber 0,39
$0,55 \mathrm{Gr}$, oder 0,3 pro 1 Kilo Katze.

4. Versuch (No. LVI). 10/III. 76. Katze von 3,53 Kilo. PferdeAleischfutterung.

A. B Int enthält 0,16 p Ot. Z. Gesammtblut $=271,0 \mathrm{Grm} .=0,433 \mathrm{Grm}$. Z.

B. Leber enthält $(118,0 \mathrm{Grm}$. $)=2,0 \mathrm{Grm}$. Z.
A. Blut 0,43
B. Leber 2,0
$2,43 \mathrm{Grm}$. Z. $=0,7$ Grm. pro 1 Kilo Katze.

5. Vexsuch (No. LIX). 13/III. 76, Katze von 3,13 Kilo. Pferdefleischfütterung.

A. 24,80 Grm. Blut geben 262,5 C.-Ctm. Decoct. 34,0 O.-Ctm. reduciren 1,0 C.-Ctm. F. L. Gesammtblut 240,0 Grm. $=0,36$ Grm. Z.

B. Leber (Decoct bis zum Schwinden der Opalescenz mit HCl gekocht) gibt $2,65 \mathrm{Grm}$. $\mathrm{Z}$. Hier wurde also ein Theil des Glykogens in Zucker übergeführt.

6. Versuch (No. LX). 15/III. 76. Katze von 2,95 Kilo. Pferdefleischfutterung.

A. 18,36 Grm. Blut geben 347,5 C.-Ctm. Decoct. 53,0 C.-Ctm. reduciren 1,0 C.-Ctm. F. L. 18,36 Grm. Blut demnach $=0,032$ Grm. $=0,17$ pCt. Z. Gesammtblut 226,0 Grm. $=0,386 \mathrm{Grm}$. Z.

B. Leber (Decoct bis zum Verschwinden der Opalescenz mit HCl gekocht) liefert 1,80 Grm. $\mathrm{Z}$.

7. Versuch (No. LXI). 16/III. 76. Katze von 3,30 Kilo. Pferdefleischfütterung.

A. 29,70 Grm. Blut geben 220,0 Decoct. 30,0 O.-Ctm. reduciren 1,0 C.-Ctm. F. L. 29,7 Grm. Blut demnach $=0,036$ Grm. $=$ 0,125 pCt. Z. Gesammtblut $=269,0 \mathrm{Grm}$. $=0,30 \mathrm{Grm}$. Z.

B. Leber gibt 1250,0 C.-Ctm. Decoct, davon 1000,0 anf 50,0 eingedampft und mit 4 Vol. Alkohol gefallt. Man findet in der ganzen Leber 1,85 Grm. Z.

8. Versuch (No. LXXXVI). Katze von 2,77 Kilo. Pferdefleischfütterung.

A. 15,63 Grm. Blut geben 260,0 C.-Ctm. Decoct. 60,0 O.-Ctm. reduciren 1,0 C.-Ctm. F. L. 15,63. Grm. Blut also $=0,021$ Grm. $=0,13$ pCt. Z. Gesammtblut $=213,0$ Grm. $=0,283$ Grm. Z.

B. Leber $(100,0$ Grm.) liefert 600,0 C.-Ctm. Decoct. 25,6 C.-Ctm. reduciren 3,0 C.-Ctm. F. L. Die Leber enthält demnach 1,38 Grm. Zucker 100,0 Decoet, mit Thierkohle behandelt geben 325,0 C.-Ctm. davon reduciren 20,6 C.-Ctm. 3,0 C.-Ctm. F. L.; demnach in der ganzen Leber 1,38 Grm. $Z$.
A. Blut 1,28
B. Leber 1,38
1,66 Grm. Z. oder 0,6 pro 1 Kilo Katze. 

fütterung.

9. Versuch (No. C). 6/IX. 76. Kater von 3,06 Kilo. Pferdefleisch-

A. 14,52 Grm. Blut geben 257,0 C.-Ctm. Decoct. 44,0 C.-Ctm. reduciren 1,0 C.-Ctm. F. L. 14,52 Grm. Blut also 0,029 Grm. Z. $=0,20$ pCt. Gesammtblut $=0,464$ Grm. Z.

B. Leber $(96,5$ Grm.) gibt 1440,0 C.-Ctm. Decoct. 50,0 Decoct, mit Thierkohle behandelt, geben 200,0 C.-Ctm.; davon reduciren 19,0 C.-Ctm. 1,0 C.-Ctm. F. L. In der Leber demnach 1,51 Grm. Z. A. Blut 0,46

B. Leber 1,51

1,97 Grm. oder 0,6 pro 1 Kilo Katze.

10. Vexsuch (No. CII.) 8/IX. 76. Kater von 3,02 Kilo. Pferdefleischfïtterung.

A. 22,78 Grm. BI ut geben 285,0 C.-Ctm. Decoct. 19,8 C.-Ctm. reduciren 1,0 C.-Ctm. F. L. 22,78 Grm. Blut also $=0,071 \mathrm{Grm}$. $=0,31$ pCt. Z. Gesammtblut $=232,0$ Grm. $=0,719$ Grm. Z.

B. Leber $(60,5$ Grm.) gibt 1310,0 C.-Ctm. Decoct (ohne Glykogen. NB. sehr fettes Thier). 50,0 C.-Ctm. Decoct, mit Thierkohle behandelt geben 292,0 C.-Ctm. Decoct; davon reduciren 25,0 C.-Ctm. 1,0 C.-Ctm. F. L. In der ganzen Leber also 1,53 Grm. Z.

A. Blut 0,719

B. Leber 1,53

2,24 Grm. Z. $=0,74$ pro 1 Kilo Katze.

11. Versuch (No. LXV). 24/III. 76. Katze von 3,63 Kilo. Pferdefleischfütterung:

Nur der Leberzucker bestimmt, nachdem das Decoct bis zum Schwinden der Opalescenz mit HCl gekocht und das aus dem Glykogen entstandene Dextrin durch Alkohol ausgefallt worden war.

Die Flïssigkeit beträgt 153,0 C.-Ctm. Davon wurden 25,0 auf 150 , und von diesen 150 wiederum 25,0 auf 100,0 C.-Ctm. verdünnt. 8,8 C.-Ctm. reduciren 3,0 C.-Ctm. F. L. Demnach in der ganzen Leber 2,16 Grm. Z.

III. Gruppe. Versuche, in denen entweder Glykogen allein, oder Glykogen und Zucker bestimmt, die Muskeln aber nicht untersucht wurden.

1. Versuch (LXVI). Katze von 2,89 Kilo. Pferdefleischfütterung. Leber 148,6 Grm. gibt 2170,0 C.-Ctm. Decoct. Die Hälfte davon gibt 3,962 Glykogen, die ganze Leber demnach 7,924 Grm. Glykogen.

2. Versuch (No. LXXXV). 14/V. 76. Katze von 2,72 Kilo. PferdeHleischfutterung.

A. 16,13 Grm. Bl ut geben 150,0 C.-Ctm. Decoct. 36,0 O.-Ctm. reduciren 1,0 C.-Ctm. F. L. 16,13 Grm. Blut also $=0,02$ Grm. $=0,12$ pCt. Z. Gesammtblut $=212 \mathrm{Grm}$. $=0,255$ Grm. Z.

B. Leber $(90,5 \mathrm{Grm}$.) gibt 2,71 Grm. Glykogen.

3. Versuch 6/IV. 75. Kater von 2,27 Kilo. Pferdefleischfiutterung. A. 38,27 Gim. Blut geben 180,0 C.-Ctm. Decoct. 19,0 C.-Ctm. reduciren 1,0 C.-Ctm. F. L. 38,27 Grm. Blut also $=0,047$ Grm. $=0,12$ pCt. Z. Gesammtblut $=172,0$ Grm. $=0,209$ Grm. Z. 
B. Leber $(59,35$ Grm.) gibt 800,0 C.-Ctm. Decoct. a) In der einen Hulfte des Decoct wird der Zucker bestimmt; man findet 0,324 Grm., also fiur die ganze Leber 0,648 Grm. Z. b) aus 400,0 C.-Ctm. Decoct erhält man 0,849 Grm. Glykogen also aus der ganzen Leber 1,698 Grm. Glykogen (=1,875 Grm. Z.)
A. Blut
0,209
B. Leber
a) 0,648
$\eta$
b) 1,875
Grm. Zucker oder 1,2 pro Kilo.

\section{Abhandlung.}

Der Fesselungsdiabetes der Katze.

Es genitgt, eine Katze auf ein Operationsbrett $\mathrm{zu}$ fesseln und zu tracheotomiren, um bei dem Thier nach circa 1/2. Stunde eine reichliche, mehrere Stunden andauernde Ausscheidung von Zucker im Harn zu veranlassen. Dieser Fesselungsdiabetes ist nach unseren Erfahrungen an mehr als 100 Versuchen eine constante Erscheinung. Wir haben ferner festgestellt, dass auch nicht tracheotomirte, sonst aber in gleicher Weise gefesselte und aufgebundene Katzen diabetisch wurden.

Die Thatsache, dass auch schon relativ geringfugige Insulten bei verschiedenen Thieren zuweilen Diabetes bewirken, ist schon mehrfach von anderen Autoren beobachtet worden. Sowohl Eckhard als auch Sehiff haben gelegentlich derartiger Befunde Erwähnung gethan, ohne ein besonderes Gewicht darauf zu legen. Als ein constantes Ergebniss des bei den meisten vivisectorischen Versuchen unentbehrlichen Fesselns und Aufbindens ist der Diabetes vor uns von Niemand beobachtet worden, was wohl dem Umstande zuzuschreiben ist, dass bisher mit Katzen uberhaupt relativ selten experimentirt wurde. In wieweit die Thatsache aber auch fur andere Versuchsthiere zutrifft, haben wir weiter zu untersuchen zunächst keine Veranlassung gehabt.

Wir mussten nun Verlauf und Intensität dieses Diabetes genauer kennen lernen. Dazu war es erforderlich die Thiere möglichst lange zu beobachten und eine zuverlässige Methode zur Aufsammlung des Harns zu besitzen. Wir fithrten zu diesem Zweck einen ca. 6 Ctm. langen, an beiden Enden geknöpften und an dem einen Ende ausserdem seitlich gefensterten silbernen Katheter ( $3-4 \mathrm{Mm}$. im Lumen) durch die Pars membranacea der Harnröhre in die Blase ein. Dies kann bei Katzen leicht mit Hilfe der kleinen Operation ausgefuhrt werden, welche $\mathrm{Naunyn}$ schon bei Kaninchenversuchen beschrieben 
hat. Man durchtrennt die Bauchhaut in der Mittellinie in einer Ausdehnung von 3-5 Cmtr. direct über der Symphyse durch einen Iongitudinalen Schnitt, spaltet hierauf die Bauchfascien, durchschneidet die Muskelansätze an der Symphyse, sucht extraperitoneal die Harnrobre auf und umgibt sie mit einer Fadenschlinge. Bei ihrem relativ langen, membranösen Verlauf kann man sie bequem wie eine Arterie auf stumpfem Hacken hervorheben, den Katheter durch einen kleinen Schnitt bis in die Blase vorschieben and wie eine Canule fest einbinden; aus seinem freien Ende tropft nun der Harn continuirlich ab und kann leicht mit Hilfe einer Glasröhrenkautschuckschlauchverbindung direct in ein calibrirtes Messgefäss geleitet werden.

Um die Dauer des Diabetes genau zu ermitteln, haben wir folgendes Verfahren eingeschlagen. $\mathrm{Da}$ in Folge der gegen das Ende des Diabetes ausnahmslos bedeutend verringerten Harnsecretion von einer Beobachtungsperiode bis zu folgenden immer kleine Mengen stark zuckerhaltigen Harns von den Wänden der Blase zurïckgehalten werden, so genitgt es nicht den Harn einfach 1 - oder $1 / 2$-stundlich zu sammeln und auf Zucker zu untersuchen; es muss auch daftr gesorgt werden, dass am Ende jeder Beobachtungsperiode die Blase völlig von dem an ihren Wänden haftenden Zucker gereinigt wird. Der wie gewöhnlich in die Harnröhre eingebundene Katheter wurde zu diesem $Z$ weck mit einer einfachen Vorrichtung verbunden, welche es ermöglichte, die Blase jeden Augenblick mit destillirtem Wasser auszuwaschen, bis das Waschwasser keine Zuckerreaction mehr gab. Das freie Ende des Katheters war durch ein Gummirohr mit dem einen horizontalen Schenkel eines T-förmigen Glasrohrs verbunden, dessen anderer horizontaler Schenkel in eine mit destillirtem Wasser geftullte Mohr'sche Bürette fubrte, während der verticale Schenkel des T-Rohrs frei, aber durch einen Quetschhahn verschliessbar nach unten in ein Messgefäss mitindete. Zwischen T-Rohr und Bürette war gleichfalls ein Quetschhahn angebracht. Von einer Stunde, resp. 1/2 Stunde zur anderen konnte sich nun, da beide Quetschbähne fuir gewölunlich geschlossen waren, der Harn in der. Blase ansammeln. Am Ende der Beobachtungsperiode liess man zuerst den Harn aus dem verticalen Ausflussrohr durch Oeffnen des Quetschhahns abfliessen, schloss denselben wieder, sobald nichts mehr abtropfte, und öffnete nun den Hahn an der Burette, so dass ein gemessenes Wasservolumen durch den horizontalen Theil des T-Rohrs und den Katheter in die Blase einströmte. Das Waschwasser wurde nach einigen Secunden wieder aus dem Abflussrohr abgelassen und nun das Aus'waschen in gleicher. Weise so oft wiederholt, bis das Waschwasser 
keinen Zucker mehr enthielt. Nur auf diese Weise waren wir sicher, das Ende der Zuckerausscheidung im Harn auf $1 / 2$ Stunde genau fixiren zu können. Bedient man sich nicht des Waschapparates, so erhält man stets fur die Dauer des Diabetes falsche, viel zu grosse Werthe. Zur Bestimmung der Dauer des Fesselungsdiabetes liegen uns im Ganzen 15 Versuche vor, die wir in Tabelle VIII iibersichtlich zusammengestellt haben.

\begin{tabular}{c|c}
\multicolumn{2}{c}{ Tabelle VIII. } \\
$\begin{array}{c}\text { Nummer des } \\
\text { Yersuchs. }\end{array}$ & $\begin{array}{c}\text { Daner des Diabetes } \\
\text { in Stunden. }\end{array}$ \\
\hline 44 & 4 \\
45 & 3 \\
46 & 7 \\
69 & 5 \\
72 & 11 \\
74 & 4 \\
77 & 3 \\
79 & 13 \\
84 & 6 \\
89 & 7 \\
140 & 8 \\
141 & $61 / 2$ \\
142 & 6 \\
143 & $5 \% / 2$ \\
144 & 6
\end{tabular}

Die durchschnittliche Dauer des Diabotes berechnet sich hiernach auf 6 Stunden $18 \mathrm{Min}^{1}$ ). Es zeigt sich, dass die zuerst mässige

1) Angaben über die Dauer des künstlichen Diabetes sind in der Literatur spürlich vertreten und selten mit besonderer Genauigkeit verzeichnet. Der Piqtare-Diabetes dauert im Allgenieinen weniger als $12 \mathrm{~h}$. (vgl. Bernard, Leçons sur la physiologie et pathologie du système nerveux. I. p. $415 \mathrm{ff}$.), nur in Ausnahmefällen scheint er sich über mehrere Tage zu erstrecken (vgl. l. c. I. p. 331). Der Diabetes, welchen Eckhardt durch Reizung des Wurms erhielt, dauerte jedenfalls länger als $3 \mathrm{~h}$. (Beiträge zur Anatomie u. Physiologie VI.) Schiff erhielt mehrmals beim Kauinchen nach Durchschneidung der Hinterstränge des Halsmarks Diabetes von $9 \frac{1}{2} \mathrm{~h}$. Dauer, beim Frosche dagegen fand er sowohl nach Piqûre als auch nach Rückenmarksdurchschneidung und -Zerstörung einen Diabetes von $4-5$ Tagen. (Schiff, Untersuchungen $a$. s. w. a. a. O. S. 74, 96, 98, dort findet sich auch die Beschreibung seines continuirlichen Diabetes). Nach der Durchschneidung des unteren Cervicalganglions endlich findet sich ein Diabetes, welcher innerhalb der zwei ersten Stunden nach der Operation seine Höhe erreicht, in 4-5 Stunden merklich abklingt und nur noch schwache Spuren binterlässt, welche sich bis $24 \mathrm{~h}$. nach dem Eingriff erhalten kömen (E c khardt, Beiträge u. s. W. IV. S. 22). Die nach Durchschneidung des ersten und zweiten Brustganglion beobachteten Zuckerausscheidungen waren nicht so intensiv. Auffallend lrurz ist jedenfalls der Curarediabetes. Eckhardt beobachtete zwischen $1^{1 / 2}-3$ h. (Beiträge IV. S. 24 ff.). Die Dauer des Kohlenoxyddiabetes hängt sehr von der Stärke der Vergiftung ab, ist oft aber ebenso kurz wie die des Curare- 
oder geringe Zuckerausscheidung rasch zu einem Maximum ansteigt, sich kurze Zeit auf demselben erhält und dann wieder ziemlich rasch abfällt. Der Beginn des Diabetes fällt meistens in den Anfang, seltener in die zweite Hïlfte der zweiten Stunde nach dem Fesseln und Aufbinden. In sehr vielen Fällen geht die Zuckerausscheidung mit starker Polyurie einher, wo dann Zucker- und Wasserausscheidung mit einander parallel steigen. Doch gehört die Polyurie keines. wegs zu den constanten Erscheinungen des Fesselungsdiabetes, ohne dass man im Stande wäre, den Grund ihres häufigen Fehlens anzugeben.

Ueber die während des Diabetes ausgeschiedenen Zuckermengen ${ }^{1}$ ) gibt Tabelle IX (auf folgender Seite) Aufschluss, in welcher 30 Beobachtungen thersichtlich zusammengestellt sind. Man ersieht aus derselben zunächst, dass auch in diesem Punkte sehr grosse Schwankungen zu Tage treten und erkennt, dass die ausgeschiedenen Zuckermengen weder zum Körpergewicht, noch zum Lebergewicht in festeren Beziehungen stehen.

Die grösste beobachtete Ausscheidung in Versuch No. 79 betrug 7,6 Grm. Zucker, die geringste in Versuch No. 1110,2 Grm. Der Durchschnitt der verscliedenen Zuckermengen berechnet sich aus obiger Tabelle auf 0,58 Grm. pro 1 Kilo Katze.

In denjenigen Fällen, welche mit starker Polyurie verbunden waren, war auch die ausgeschiedene Zuckermenge am grössten. Der Ermährungszustand der Thiere liess keinen sehr ausgesprochenen Einfluss auf die ausgeschiedenen Zuckermengen erkennen. Fette wie magere Thiere verloren bald grössere bald geringere Zuckermengen. Während man sonst gewöhnlich den künstlichen Diabetes im Hungerzustande nicht zu erzeugen vermag, haben wir ihn auch bei 3 Katzen beobachtet, welche 3 , resp. 7 und 8 Tage absolut gehungert hatten, wenn auch in diesen Fällen nux geringe Mengen Zuckers im Harne auftraten.

Die Frage, ob nach eingreifenderen Operationen, z. B. Rücken-

diabetes (Senff, Dissert. Dorpat 1865). Amylnitrit wirkt gleichfalls nur für einige, höchstens 12 Stunden (Sebold, Dissert. Marburg 1874). Der Diabetes, weIcher durch Einleiten 1 proc. Kochsalz- und anderer verdünnter Lösungen entsteht, dauert im Allgemeinen so lange wie die Injection oder bis zum Tode des Thieres (K îlz, Eckhardt's Beiträge); es scheint aber, dass sehr langes Durchleiten zuletzt das Vermögen der Zuckerausscheidung erschöpft; jedenfalls dauert dies aber beim Kaninchen mindestens 12 Stunden.

1) Die Zuckerbestimmung im Harn führten wir nach der Fehling'schen Methode aus und controlirten das erhaltene Resultat durch die Circumpolarisation des Harns im Cornu'schell Apparat (vgl. dies. Arch. Bd. VII. S, 491). 
Tabelle IX.

\begin{tabular}{|c|c|c|c|c|c|}
\hline 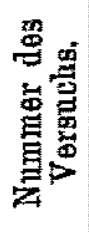 & $\begin{array}{l}\text { Körporgewicht } \\
\text { in Kilogrm. }\end{array}$ & $\begin{array}{l}\text { Lebergewicht } \\
\text { in Grm. }\end{array}$ & $\begin{array}{l}\text { Zuokerverlust } \\
\text { des Thieres } \\
\text { wälnend } \\
\text { des Diabetes } \\
\text { in Grm. }\end{array}$ & $\begin{array}{c}\text { Zuckervorlust } \\
\text { pro } \\
1 \text { Kilo Katze. }\end{array}$ & $\begin{array}{l}\text { Zuckerverlust } \\
\text { in pCt. } \\
\text { des } \\
\text { Lebergewichts. }\end{array}$ \\
\hline $\begin{array}{r}34 \\
41 \\
44 \\
45 \\
46 \\
48 \\
72 \\
79 \\
81 \\
104 \\
106 \\
108 \\
109 \\
110 \\
111 \\
112 \\
113 \\
114 \\
117 \\
118 \\
105 \\
140 \\
141 \\
149 \\
143 \\
144 \\
89 \\
69 \\
74 \\
77\end{array}$ & $\begin{array}{l}\overline{1,72} \\
3,90 \\
3,75 \\
2,50 \\
3,10 \\
2,60 \\
3,77 \\
2,46 \\
3,12 \\
2,58 \\
2,90 \\
2,70 \\
2,65 \\
3,35 \\
2,64 \\
2,64 \\
2,84 \\
2,60 \\
2,40 \\
2,72 \\
4,07 \\
3,83 \\
2,55 \\
2,20 \\
2,75 \\
2,10 \\
3,6 \\
3,21 \\
3,02\end{array}$ & $\begin{array}{r}- \\
- \\
- \\
\overline{-} \\
52,9 \\
109,5 \\
81,0 \\
73,0 \\
76,0 \\
78,0 \\
84,0 \\
63,3 \\
72,0 \\
-1 \\
83,0 \\
84,0 \\
7 \\
75,0 \\
107,0 \\
114,0 \\
83,0 \\
82,7 \\
82,0 \\
80,3 \\
139,5 \\
87,5 \\
112,1\end{array}$ & $\begin{array}{l}2,26 \\
0,94 \\
0,61 \\
0,69 \\
1,50 \\
0,74 \\
3,0 \\
7,6 \\
2,05 \\
1,59 \\
1,30 \\
1,90 \\
1,40 \\
1,31 \\
0,20 \\
1,36 \\
2,10 \\
0,79 \\
1,35 \\
1,80 \\
0,48 \\
4,43 \\
1,80 \\
1,69 \\
0,51 \\
3,40 \\
1,35 \\
3,3 \\
1,96 \\
1,26\end{array}$ & $\begin{array}{l}0 \overline{0,54} \\
0,15 \\
0,18 \\
0,60 \\
0,24 \\
1,10 \\
2,0 \\
0,53 \\
0,50 \\
0,50 \\
0,65 \\
0,51 \\
0,49 \\
0,05 \\
0,51 \\
0,78 \\
0,28 \\
0,51 \\
0,75 \\
0,17 \\
1,0 \\
.0,47 \\
0,66 \\
0,23 \\
1,2 \\
0,67 \\
0,91 \\
0,61 \\
0,41\end{array}$ & $\begin{array}{l}- \\
- \\
- \\
- \\
\overline{5,6} \\
6,9 \\
2,5 \\
2,1 \\
1,7 \\
2,5 \\
1,6 \\
2,0 \\
\overline{1,8} \\
0,9 \\
1,6 \\
\overline{0,6} \\
4,0 \\
1,5 \\
2,0 \\
0,6 \\
4,1 \\
1,6 \\
2,3 \\
2,2 \\
1,1\end{array}$ \\
\hline
\end{tabular}

marksdurchschneidungen, der Diabetes längere Zeit dauert und einen grösseren Zuckerverlust verursacht als nach einfacher Fesselung, können wir dahin beantworten, dass sowohl nach Verlauf als nach Intensität kein deutlicher Unterschied zu erkennen ist. Die Arbeiten der Autoren tuber den künstlichen Diabetes enthalten keine näheren Angaben über die ausgeschiedenen Zuckermengen, so dass ein Vergleich in dieser Hinsicht mit unseren Ergebnissen nicht angestellt werden kann. ')

1) Ueber das Quantum Zucker, welches Thiere durch einen künstlichen Diabetes verlieren, finden wir in der Literatur keine genügenden Nachweise; nur hie und da ist der Procentgehalt des Diabetesharns an Zucker ermittelt worden. Aus einigen Experimenten von $\mathrm{K}$ ù $\mathrm{tz}$ el (Experimentelle Beiträge z. Lehre v. d. Melliturie (Dissert. Berlin 1872) kann man allenfalls die Gesammtzuckerausscheidung berechnen, wie in Experiment 2, wo $0,29 \mathrm{Grm}$. nach Injection von 1 proc. Lösung von phosphorsaurem Natron ausgeschieden wurden. - Bernard hat über 
Angesichts der relativ bedeutenden Zuckerverluste, welche die Thiere durch den Fesselungsdiabetes erlitten, mussten wir die Möglichkeit zugestehen, dass durch diesen Diabetes allein der Glykogenvorrath der Leber so bedentend reducirt würde, dass man nach keinem weiteren Erklärung:sgrunde für das schliessliche völlige Verschwinden der Kohlehydrate zu suchen brauchte. Es gab, um hieritber ins Klare zu kommen, nur den einen Weg, in mehreren Versuchen die Thiere unmittelbar nach dem Ablauf des Diabetes zu tödten und den Kohlehydratbestand ihres Körpers quantitativ zu bestimmen.

Tabelle $\mathrm{X}$ fasst die Resultate von 7 zu diesem Zweck angestellten Versuchen zusammen, deren ausfthrliche Protokolle diesem Abschnitt als Anhang beigegeben sind.

Wir ersehen, dass trotz sehr erheblicher Zuckerverluste im Diabetes der Kohlehydratbestand der nach dem Diabetes getödteten Thiere nicht auffallend geringer ist als der ganz normaler Thiere.

Tabelle $\mathrm{X}$.

\begin{tabular}{|c|c|c|c|c|c|c|c|c|c|c|c|}
\hline \multirow[b]{2}{*}{ 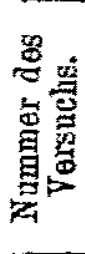 } & \multirow[b]{2}{*}{ 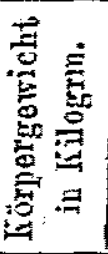 } & \multirow[b]{2}{*}{$\begin{array}{c}\text { fErnäbrungs- } \\
\text { zustand. }\end{array}$} & \multirow[b]{2}{*}{ 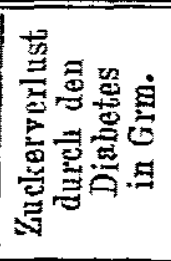 } & \multirow[b]{2}{*}{ 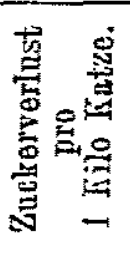 } & \multicolumn{7}{|c|}{ Fohlehydratbestind nach dem Ablanf a. Wiabetes: } \\
\hline & & & & & 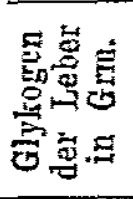 & 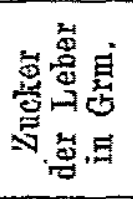 & 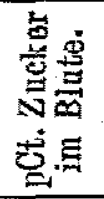 & 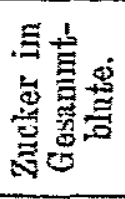 & 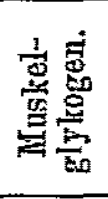 & 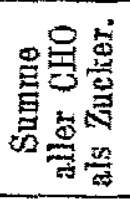 & 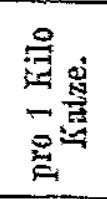 \\
\hline & & & & & & & & & & & \\
\hline 14 & 3,83 & ehr. & & 0 & 1,8 & 2,0 & 0,2 & & 0 & & \\
\hline 14 & 2,55 & Iässi & & & 2 & 1,06 & $0, \%$ & 0 & 1,22 & 5 & 2, \\
\hline 14 & 2,2 & $\mathrm{M}$ & & & 3, & 1,10 & 0 , & 0 , & 0,2 & & 2 \\
\hline 14 & 2,75 & Mässig fett. & & & & $1,0(?)$ & & 0, & 0,44 & 5,84 & 2,1 \\
\hline 8 & & 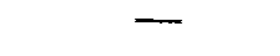 & & & & -2 & & & & - & \\
\hline 89 & & & 1,35 & 0,64 & & 0,98 & & 0,40 & 0,0 & 3,07 & 1,4 \\
\hline
\end{tabular}

Wir fügen dieser Tabelle ausserdem noch die Bemerkung bei, dass die zu dieser Versuchsreihe beniitzten Katzen, namentlich die von No, 140 und 141 zufullig ausnehmend fett waren, woraus zu vermuthen ist, dass ibmen schon von vorneherein geringe Glykogenvorräthe zu Gebote standen. Die Versuche liefern jedenfalls den directen Beweis, dass der durch den Diabetes bedingte Znckerverlust nicht als Erklürung des im folgenden Abschnitte eingehend besprochenen Verschwindens der Kohlehydrate nach allmählicher $A b$ kühlung herangezogen werden kann. Ueber den Zuckergehalt des

eine Piqûre an der Katze referirt (Leçons sur la physiologie et pathologie du syst. nerv. I. p. 432), welche von einem auffallend holen Zuckergehalt des Urins gefolgt war ( 4 pCt.). Dass sich Kaninchen aber ganz anders verhalten müssen wie Katzen, geht aus dem Versuche hervor, wo Bermard die Piqure-Wirlung verhindert, indem er das Halsmark durchschneidet. 
Blutes während des Diabetes liegen uns nur 3 Bestimmungen vor. In allen 3 war der Blutzuckergehalt auffallend wenig tiber die Norm gesteigert, nicht so bedeutend, als man nach den Angaben von Becker ${ }^{1}$ ) und Bernard hätte erwarten sollen. In dem einen Falle enthielt das Blut 0,2 pCt., in dem anderen $0,26 \mathrm{pCt}$. und eine Stunde später anf der Höhe des Diabetes 0,34 pCt. Zncker. Tabelle $\mathrm{X}$ zeigt, dass nach dem Authören des Diabetes das Blut in der Regel wieder die normale Menge Zucker enthält.

Der Eingriff, durch welchen die im Vorstehenden näher besprochene Form des kinstlichen Diabetes hervorgerufen wird, - die Fesselung: - ist ein ziemlich complicirter. Er trifft nicht ein einzelnes Organ oder eine bestimmte Stelle des Körpers sondern dessen Gesammtheit, und es ist von vorneherein wenig Aussicht vorhanden, unter den manchfaltigen Störungen, denen das g'efesselte Thier unterworfen ist, diejenige herauszufinden, welche die eigentliche Diabetesexzeugende Ursache ist.

Wenn wir nun auch der Meinung sind, dass ein erfolgreiches Studium des Wesens der künstlichen Glykosurie bei dem gegenwärtigen Stande unserer Kenntnisse nicht möglich ist und die ganze Frage iiberhaupt ausserhalb des Bereichs unserer Untersuchungen liegt, so haben wir doch, soweit es möglich war, versucht, die bei der Fesselung mitwirkenden einzelnen Hauptmomente zu analysiren und wollen in Kirze die gewonnenen Resultate mittheilen.

Die Fesselung eines Thieres in Verbindung mit der Tracheotomie lässt sich ungezwungen in folgende Factoren zerlegen:

1. die durch das Aufbinden und die Tracheotomie bedingte $A b$ kithlung;

2. die zahlreichen sensibeln Reize (Schmerzen), welche mit der Festschnirung der Extremitäten, dem Knebeln und den blutigen Operationen (Tracheotomie) verbunden sind;

3. die Circulati s sooxungen, welche die oben aufgezählten Eingriffe und die veränderte Haltung des Thieres mit sich bringt.

Es liess sich zunächst leicht durch den Versuch feststellen, dass die durch dass Fesseln und Tracheotomiren des Thieres verursachte Abkihlung nicht die alleinige Diabetesursache ist. In einer Reihe von Versuchen wurden die gefesselten Thiere nicht tracheotomirt und ausserdem durch dicke Umhillungen von Pelz and Watte vor der Abkiihlung geschitzt. Anch bei diesen Thieren erfolgte, wenn auch häufig etwas später, eine reichliche Glykosurie die weder nach

1) Zeitschrift für wissensch. Zoologie. 1853. 
Intensität noch nach Dauer von der verschieden war, welche die sich stark abkuhlenden Thiere acquirirten.

Durch andere Versuche erfuhren wir allerdings, dass bei sehr energischer Ablühlung, wenn sie ohne alle weiteren Eingriffe durch häufig wiederholte Eiswasserbäder bewirlst wird, wenn nicht constant so doch in einzelnen Fällen Glylrosurie entsteht. Die einschlägigien Versuche werden in einer der folgenden Abhandlungen im Detail mitgetheilt werden. Es ist also zunächst constatirt, dass Ablithlung allein fur sich bei Katzen zum Diabetes führen kann, dass aber diese pathologische Erscheinung beim Fesselungsversuche auch ohne ihre Mitwirkung zu Stande kommt.

Was die sensibeln Reize, die Schmerzen betrifft, welche unsere Versuchsanordnung mit sich bringt, so waren sie durch keine Modification des Verfahrens zu umgehen. Die Anwendung von Narcoticis verbot sich von selbst; denn es ist bekannt, dass sowohl Morphium als auch Chloroform und Chloral häufig genug fur sich allein Zuckerausscheidung im Harn bewirken.

Es konnte aber der Weg eingeschlagen werden, bei den Versuchsthieren einen möglichst isolirten sensibeln Reiz zu appliciren und zuzusehen, ob auch er von Diabetes gefolgt sei. Wir durchschnitten zu diesem Zwecke ungefesselten mit der Hand auf dem Operationstisch festgehaltenen Thieren möglichst rasch einen oder beide Nervi ischiadici. Ueber die Resultate geben nachstehende Versuchsdaten Aufschluss.

Versuch XXIV. Kräftige Katze. Vor der Operation wird viel klarer Harn -entleert, der frei von Eiweiss und Zucker ist. Von $10 \mathrm{~h} .52 \mathrm{~m}$. bis $10 \mathrm{~h} .56 \mathrm{~m}$. beide $\mathrm{Nu}$. ischiadici durchsehnitten.

$11 \mathrm{~h} 26 \mathrm{~m}$ Einige Tropfen Harn gewonnen, welche mit Kalilauge gekocht sich deutlich gelb färben.

11 h 56 m 2 C.-Ctm. Harn gewonnen, welche reichlich Zucker enthalten.

$12 \mathrm{~h} 26 \mathrm{~m} 3$ C.-Ctm. Harn, stark zuckerhaltig

12 h $56 \mathrm{~m}$ wenig Harn: entwickelt mit Hefe angesetzt im Eudiometer bis zum anderen Morgen reichlich Kohlensäure.

Das Thier wird nun in einen Käfig gesetzt. Am anderen Morgen finden sich 40,0 O.-Ctm. trüben Harns gesammelt worin sich grosse Mengen Zucker befinden. Der jetzt mit dem Katheter dem Thier entnommene Hain ist zuckerfrei.

Versuch XXV. Die gleiche Katze wird 4 Tage nach dem ersten Versuche wieder vorgenommen; der Harn zuckerfrei befunden. $11 \mathrm{~h}$. $15 \mathrm{~m}$. werden die centralen Stümpfe beider $\mathrm{Nn}$. ischiadici oberhalb der ersten Durchschneidungsstelle noch einmal durchschnitten.

12 h 15 m Harn zuckerhaltig. 
$1 \mathrm{~h} 15 \mathrm{~m}$ desgleichen.

$4 \mathrm{~h}-\mathrm{m}$ desgleichen; eine Probe des Harns mit Hefe im Eudiometer aufgestellt, entwickelt reichlich Kohlensäure.

Diese Versuche zeigen, dass ein starker sensibler Reiz allein schon hinreicht, bei der Katze Diabetes zu erzeugen. Die Operation wurde in beiden Fällen so rasch ausgefuhrt, dass wir wohl annehmen duirfen, bei dem Thiere keine anderen den Versuch complicirenden Eingriffe verursacht zu haben. Wir müssen allerdings hinzufügen, dass wir auch Ischiadicus-Durchschneidungen ausgefiuhrt haben, auf welche kein Diabetes folgte, dass daher diese Methode ebenso wenig. wie die einfache Abkühlung unfehlbar zur Glykosurie fthrt. Es scheint namentlich, dass sehr starke Thiere schwerer diabetisch werden als schwächere.

Fur uns ist es indessen genug, zu wissen, dass der Diabetes in Folge schmerzhafter Eingriffe entstehen kaun; er wird natürlich nach denselben um so sicherer sich einstellen, wenn noch andere Momente hinzukommen, welche wie z. B. die Abkühlung gleichfalls in die Kategorie der Diabetes erzeugenden Ursachen gehören.

Eine isolirte Erzeugung von Circulationsstörungen ähnlich denen, welche das Fesseln bedingt, ist nicht müglich ohne gleichzeitige sensible Reizungen. Es konnte daher über die Betheiligung dieses Momentes bei der Entstehung des Diabetes von uns nichts Näheres ermittelt werden. Es ist übrigens bekannt genug, dass Circulationsstörungen bei verschiedenen Thieren Diabetes erzeug'en können, so futhrt die Unterbindung der Aorta bei Kaninchen stets zur Glykosurie (und Kaninchen werden viel schwerer diabetisch als Katzen). Unter Anderen hat auch S $\mathrm{ch}$ iff ${ }^{1}$ ) beobachtet, dass die Unterbindung: der Hauptgefässstämme einer Extremität bei einer Katze Zuckerausscheidung im Harn hervorrief.

Dieser Befund kommt dem unsrigen schon sehr nahe. 2) Es ist ibrigens dagegen einzuwenden, dass die Erzeugung derartiger Circulationsstörungen niemals ohne gleichzeitige starke sensible Reize möglich ist.

Das Vorstehende diufte gentigen um nachzuweisen, dass in der Anordnung des Fesselns der Thiere nicht nur eine, sondern eine

1) Journ. de l'anatomie et de la physiologie. 1866.

2) Schiff hat auch folgendes uns interessirende Experiment gemacht: Nous avons fortement lié en masse la cuisse d'un animal de manière a interrómpre complètement la circulation et nous l'avons laissé ainsi une demi-heure une heure ou deux heures. Nous avons produit ainsi une glycosurie qui durait jusqu’à 12 heures. 
ganze Reihe von Ursachen enthalten ist, welche eine Störung im Zuckerstoffwechsel im Gefolge haben können. Diese Erkenntniss füht uns aber leider zu keinem aufgeklärteren Standpunkt hinsichtlich des Wesens der experimentell erzeugten Glykosurie. Wir haben wohl Ursachen furr dieselbe aufgefunden. Dieselben sind aber so allgemeiner Natur, dass bei näherer Ueberlegung jede weitere Discussion über die Entstehungsweise der Glykosurie miissig erscheinen muss.

Abkiihlung, Schmerzen, Circulationsstörungen sind Einflitsse, welche ausser dem Diabetes noch eine lange Reihe theils bekannter, theils dunkler Veränderungen im Gefolge haben, ganz abg'esehen davon, dass eine sensible Reizung oder auch eine Abkuhlung stets auch Circulationsstörungen erzeugen, und Circulationsstörungen ihrerseits wieder sowohl Abktihlung als auch Schmerzen verursachen können.

Man wird aber nur um so bereitwilliger anf weitere Speculationen verzichten, wenn man vollends bedenkt, wie wenig Sicheres iiber die complicirte Mechanik der Leberfunctionen bekannt ist, ein Gebiet wo jeder Schritt vorwärts durch eine nnentschiedene Controverse gehemmt ist. Hier liegen unserer Meinung nach fiur jetżt die Angriffspunkte für die weitere experimentelle Forschung. In die Frage vom ktinstlichen Diabetes wird dann schon von selbst mehr Klarheit kommen.

Bei unseren weiteren Untersuchungen hatte der Fesselungsdiabetes lediglich die Bedeutung einer accidentellen Erscheinung, welche nur so lange bertucksichtigt werden musste, bis es nachgewiesen war, dass der durch dieselbe bedingte Zuckerverlust keinen wesentlichen Einfluss auf den Kohlehydratbestand des Körper's äussert. Es muss der Zukunft anheimgegeben werden, einen Zusammenhang des Diabetes mit den iibrigen von uns beobachteten Anomalien im Kohlehydratstoffwechsel, welche durch die Wärmeentziehung geschaffen werden, aufzudecken. Heute stehen sich beide Reihen von Erscheinungen noch völlig: unvermittelt g'egentiber.

\section{ANHANG.}

Protokolle und analytisohe Belege.

Versuch No. LXXXIX. 20/V. 76. Katze von 2,1 Kilo. Rindfleischfütterung.

Aufgebunden 7 h. frilh; Tracheotomie, Cystotomie, Präparation einer Carotis, alles fertig um $7 \mathrm{~h} .50 \mathrm{~m}$. 
Zeit. Temp. in ano. Harnsecretion.

\begin{tabular}{|c|c|c|c|c|c|c|c|c|}
\hline $8 \mathrm{~h}$ & 38,7 & & & & & & & \\
\hline $9 \mathrm{~h}$ & 37,8 & 9,5 & C. - Ctm. & Harn & mit & 0,38 & Grm. & Z. \\
\hline $10 \mathrm{~h}$ & $\begin{array}{l}37,4 \\
37,4\end{array}$ & 9,5 & $n$ & $n$ & $\pi$ & & 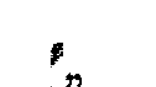 & \\
\hline $\begin{array}{ll}1 & \mathrm{~h} \\
2 & \mathrm{~h}\end{array}$ & $\begin{array}{l}37,4 \\
37,5\end{array}$ & & & & $n$ & & & \\
\hline $1 \mathrm{~h}$ & 37,7 & 9,5 & $n$ & $n$ & $n$ & 0,49 & $n$ & $n$ \\
\hline $2 \mathrm{~h}$ & 37,7 & 6,5 & $n$ & & & 009 & & \\
\hline $3 \mathrm{~h}$ & 37,5 & & $n$ & $n$ & $\pi$ & 0,00 & $n$ & $n$ \\
\hline $4 \mathrm{~h}$ & - & 3,0 & $n$ & $n$ & olne & $\mathrm{Z}$ & & \\
\hline $5 \mathrm{~h}$ & 37,0 & 3,0 & $n$ & $n$ & $n$ & $n$ & & \\
\hline $6 \mathrm{~h}$ & 36,9 & 2,5 & $n$ & $n$ & $n$ & $n$ & & \\
\hline $7 \mathrm{~h}$ & 36,5 & 2,5 & $n$ & $n$ & $n$ & $\eta$ & $\begin{array}{l}\text { Das } 7 \\
\text { Verblu }\end{array}$ & $\begin{array}{l}\text { Thier wird durch } \\
\text { atung getodtet. }\end{array}$ \\
\hline
\end{tabular}

Die chemische Untersuchung beginnt sofort.

A. 28,70 Grm. Blut geben 250,0 C.-Ctm. Decoct mit 0,25 pCt. Z. Gesammtblut $=0,40$ Grm. Z.

B. Le ber (80,3 Grm.) gibt 1450 C.-Ctm. Decoct. a) 100,0 C.-Ctm. Decoct mit Thierkohle behandelt geben 265,0 C.-Ctm., wovon 19,4 C.-Ctm. 1,0 C.-Ctm. F. L. reduciren. Demnach sind in der ganzen Leber 0,986 Grm. Z. b) 1251,0 G.-Ctm. Decoct liefern 1,17 Grm. Glykogen, die ganze Leber also $1,35 \mathrm{Grm}$. $=1,69$ Grm. Z.).

C. Muskeln enthalten keine wägbaren Mengen Glykogen.

D. Im Harn werden im Ganzen ausgeschieden 1,53 Grm. Z. oder 0,64 pro 1 Kilo Katze.
A. Blint 0,40
B. Leber
a) 0,98
$"$
b) 1,69
3,07 Grm. oder 1,4 pro 1 Kilo Katze.

Versuch CXI. 18/II. 77. Starker Kater von 4,07 Kilo. Rindfleischfütterung. Anordnung wie im vorigen Versuch; ausserdem Waschapparat zum Auswaschen der Blase. Vor dem Aufbinden in ano $38,50 \mathrm{C}$. Zeit. Temp, in ano.

$9 \mathrm{~h} \quad 37,0$

$10 \mathrm{~h} \quad 37,0$

11 h 37,2

30,0 C.-Ctm. Harn abgelassen.

$12 \mathrm{~h} \quad 37,5$

10,0 zuckerhaltig.

$1 \mathrm{~h} \quad 37,8$ Blase 4 mal gewaschen. IV. Waschwasser noch zucker-

$2 \mathrm{~h} \quad 37,8$ haltig.

$3 \mathrm{~h} \quad 37,6$

$4 \mathrm{~h} \quad 37,3$

$5 \mathrm{~h} \quad 37,1$ Harn und Waschwässer frei von Zucker.

5 h $30 \mathrm{~m}$ Das Thier wird durch Verbluten getödtet.

Section und chemische Untersuchung sofort vorgenommen. Ungemein reichliches Fettg'ewebe. 
A. 26,20 Grm. Blut geben 215,0 C.-Ctm. Decoct. 13,0 C.-Ctm. reduciren 1,0 O.-Ctm. F. L. also in 26,20 Grm. Blut 0,082 Grm. $=0,31$ pCt. Z. Gesammtblut $=313,0$ Grm. $=0,97$ Grm. Z.

B. Leber (107,0 Grm.) gibt 1060,0 C.-Ctm. Decoct ohne Glykogen. Nach dem Enteiweissen durch Alkohol anf Zucker titrirt; in der ganzen Leber sind 1,32 Grm. $Z$.

C. Muskeln frei von Glykogen.

D. Im Harn werden im Ganzen 4,43 Grm. Z. ausgeschieden.

$$
\begin{aligned}
& \text { A. Blut } \frac{0,97}{\text { B. Leber } 1,32} \\
& 2,29 \text { Grm. oder } 0,56 \text { pro } 1 \text { Kilo Katze. }
\end{aligned}
$$

Daner des Diabetes 8 Stunden.

Versuch CXLT. 21/Ir. 77. Kater von 3,83 Kilo. Rindfleischfütterung. Anordnung wie im vorhergehenden Versuch. Temperatur in ano vor dem Aufbinden $38,5^{\circ} \mathrm{C}$. Alle Operationen beendet $1 \mathrm{~m} 8 \mathrm{~h} .15 \mathrm{~m}$. früh. Zett. Temp. in ano.

$\begin{array}{rrr}9 & \mathrm{~h} & 38,5 \\ 10 & \mathrm{~h} & 38,4 \\ 11 & \mathrm{~h} & 38,2 \\ 12 \mathrm{~h} & 38,0 \\ 1 \mathrm{~h} & 37,8 \\ 2 \mathrm{~h} & 37,8\end{array}$

1 lh $30 \mathrm{~m}$ IV. Waschwasser zuckerfrei.

2 h $30 \mathrm{~m}$ Harn und Waschwässer zuckerfrei. Das Thier wird dureh Verbluten getödtet; Section und cliemische Untersuchung sofort vorgenommen:

A. 26,85 Grm. B1 ut geben 220,0 C.-Ctm. Decoet. 18,6 C.-Ctm. reduciren 1,0 C.Ctm. F. L.; sonach in 26,85 Grm. Blut 0,059 Grm. $=0,22$ pCt. Z. Gesammtblut $294,0 \mathrm{Gr}$. $=0,64 \mathrm{Grm}$. Z.

B. Leber $(114,0$ Grm.) gibt 2700 C.-Ctm. Decoct. a) nach dem Enteiweissen reduciren 20,0 C.-Ctm. 3,0 C.-Ctm. F. L., demnach in der ganzen Leber 2,0 Grm. Z. b) 2600,0 C.-Ctm. Decoct liefern 1,75 Grm. Glylkogen, die ganze Leber 1,81 Grm. ( 2,0 Grm. Z.). Bemerk. Es hat sich nachträglich horausgestellt, dass dio Leber nicht völlig erschöpft war und der Rückstand noch ziemlich viel Glykogen enthielt; die Zahl für den Glykogengehalt der Leber ist demnach erheblich $\mathrm{z} \mathrm{n} \mathrm{kl}$ ei $\mathrm{n}$.

C. Muskeln frei von Glylkogen.

D. Harn. Sämmtlicher Harn mit den Waschwässern vereinigt, nach dem Enteiweissen 127,0 C.-Ctm. Davon 10,0 C.-Ctm. ad 100,0 C.-Ctm. verdünnt. 17,2 O.-Ctm. reduciren 5,0 C.-Ctm. F. L. Im ganzen Harn also 1,8 Grm. Z. $(0,47$ pro 1 Kilo Katze).

A. Blut 0,64

B. Leber a) 2,00

$$
\text { ". b) } 2,00
$$

Daner des Diabetes $61_{/ 2}$ Stunden.

Versuch No. CXLII. 22/II. 77. Katze vọn 2,55 Kilo. Rindfleischfutterung. .Versuchsanordnung wie vorher. Temperatur in ano vor dem Aufbinden $38,1^{\circ} \mathrm{C}$. Alle Operationen beendet $\operatorname{um} 8 \mathrm{~h} 15 \mathrm{~m}$ früh. 
Zeit. Temp. in ano.

9 h 36,9

$11 \mathrm{~h} \cdot 36,1$

$12 \mathrm{~h} \quad 36,3$

$1 \mathrm{~h} \quad 36,4$

2 h 36,2

Harn und sämmtliche Waschwässer zuckerfrei. Das Thier wird daher durch Verbluten getodtet. Section und chemische Untersuchung sofort vorgenommen.

A. 26,54 Grm. Blut geben 155,0 C.-Ctm. Decoct. 26,0 C.-Ctm. reduciren 1,0 C.-Ctm. F. L., also 26,54 Grm. Blut $=0,06$ Grm. 0,23 pCt. Z. Gesammtblut $=196,0$ Grm. 0,45 Grm. Z.

B. Le ber $(83,0$ Grm.) gibt 2540,0 C.-Ctm. Decoct. a) nach dem Enteiweissen durch Alkohol reduciren 12,0 C.-Ctm. 1,0 C.-Ctm. F. L. Die Leber enthält daher 1,06 Grm. Z. b) 2440,0 G.-Ctm. Decoct liefern 2,08 Grm., die ganze Leber 2,13 Grm. Glykogen $=2,36$. Grm. Z.).

C. M uskeln 207,0 Grm. geben 0,248 Grm. Glykogen; die Gesammtmuseulatur $=2 / 5$ K. Gew. $=1,22$ Grm. Glykogen $(=1,35$ Grm. Z.).

D. Harn. Sämmtlicher Harn mit den zuckerhaltigen Waschwässern vereinigt, nach dem Enteiweissen 115,0 C.-Ctm. 10 C.-Ctm. ad 100,0 C.-Ctm. verdünnt. Davon reduciren 17,0 O.-Ctm. 5,0 O.-Ctm. F. L. Im Harn demnach $1,69 \mathrm{Grm}$. Z. ausgesehieden (oder 0,66 pro 1 Kilo).

A. Blut 0,45

B. Leber a) 1,06

b) 2,36

C. Muskeln 1,35

5,22 Grm. oder 2,0 Grm. pro 1 Kilo. Katze.

Dauer des Diabetes 6 Stunden.

Versuch No. CXLIII. 24/II. 77. Katze von 2,2 Kilo. Rindfleischfütterung. Anordnung wie vorher. Temperatur in ano vor idem Aufbinden? Alle Operationen beendet $8 \mathrm{~h} .10 \mathrm{~m}$.

Zeit. Temp. in ano.

8 h $30 \mathrm{~m} \cdot \quad 37,0$

$9 \mathrm{~h}-\mathrm{m} .35,7$

$10 \mathrm{~h}-\mathrm{m} \quad 35,6$

$11 \mathrm{~h}-\mathrm{m} \quad 36,0$

$12 \mathrm{~h}-\mathrm{m} \quad 36,6$

$1 \mathrm{~h}-\mathrm{m} \quad 36,0 \quad 1 \mathrm{~h} .30 \mathrm{~m}$. Harn und Waschwässer znckerfrei. Das Thier wird daher durch Verbluten getödtet. Section und chemische Untersuchung sofort vorgenommen; wenig Fettgewebe.

A. 32,81 Grm. Blut geben 240,0 C.-Ctm. Decoct. 32,5 C.-Ctm. reduciren 2,0 C.-Gtm. F. L., also in 32,81 Grm. Blut 0,073 Grm. $=0,22$ pCt. Z. Gesammtblut 169,0 Grm, $=0,37$ Grm. Z.

B. Leber (82,7 Grm.) liefert 5000,0 C.-Ctm. Decoct. a) Nach dem Enteiweissen reduciren 17,0 O.-Ctm. 3,0 C.-Ctm. F. L. Die Leber enthält demnach 1,1 Grm. Z. b) 4800,0 G.-Ctm. geben 3,825 Grm. Glykogen, die ganze Leber 3,9 Grm. (= 4,3 Grm. Z.). 
308 XVI. R. Bogers u. F. A. HoFFMAN Kohlehydratstoffwechsel.

C. M uskeln. 234,0 Grm. geben 0,076 Grm. Glykogen, die Gesammtmuskeln $(880,0$ Grm.) 0,27 Grm. Glykogen (=0,32 Grm. Z.).

D. Harn. Sümmtlicher Harn mit don zuckerhaltigen Waschwässern vereinigt, nach dem Enteiweissen 45,0 C.-Ctm. 10,0 C.-Ctm. ad 100,0 O.-Ctm. verdtinnt. Davon reduciren 21,8 C.-Ctm. 5,0 C.-Ctm. F. L. Im Harn wurden also 0,51 Grm. Z. ausgeschieden (oder 0,23 Gr'm. pro Kilo Katze).
A. Blut
0,37
B. Leber a) 1,10
C. Muskeln 0,32
b) 4,40
6,19 Grm. oder 2,8 pro 1 Kilo Katze.

Dauer des Diabetes $5^{1 / 2}$ Stunden.

Yersuch No. CXIIV. 25/II. 77. Kater von 2,75 Kilo. Rindfleischfiitterung. Anordnung wie vorher. Temperatur in ano vor dem Aufbinden $38,6^{\circ} \mathrm{C}$. Alle Operationen beendet $8 \mathrm{~h}$. frith.

Zeit. Temp. in ano.

$\begin{array}{lll}9 & \text { h } & 36,7\end{array}$

$10 \mathrm{~h} \quad 36,9$

$11 \mathrm{~h} \quad 37,6$

$12 \mathrm{~h} \cdot 37,5$

$1 \mathrm{~h} \quad 37,5$

2 h 37,5 Harn und sämmtliche Waschwässer zuckerfrei. Das Thier wird daher verblutet, die Section und die chemische Untersuchung sofort vorgenommen.

A. 25,5 Grm. Blut geben 260,0 C.-Ctm. Decoct. 22,8 C.-Ctm. redueiren 1,0 C.-Ctim. F. L., also in 25,5 Grm. Blut 0,057 Grm. $=$ 0,22 pCt. Z. Gesammtblut 211,0 Grm. = 0,46 Grm. Z.

B. Le ber $(82,0$ Grm.) gibt 3100 O.-Ctm. Decoct. a) (die Zuckerbestimmung missgliuckt). b) 3000,0 Decoct geben $3,14 \mathrm{Grm}$., die ganze Leber also 3,52 Grm. Glykogen $=(3,90$ Grm. Z.).

C. MuskeIn. 324,5 Grm. enthalten 0,13 Grm. Glykogen. Die Gesammtmusculatur $=1100,0$ Grm., also 0,44 Grm. Glykogen (= 0,48 Grm. Z.).

D. Harn. Sämmtlicher Harn, mit den zuckerhaltigen Waschwässern vereinigt, nach dem Enteiweissen 150,0 C.-Ctm. Es wurden 10,0 ad 100,0 verdiunt; davon reduciren 11,0 C.-Ctm. 5,0 C.-Ctm. F. L. Im Harn sind daher ausgeschieden $3,4 \mathrm{Grm}$. Z. oder 1,2 pro 1 Kilo Katze.
A. Blut
0,46
B. Leber a) 1,00 (berechnet)
C. Muskeln 0,48
b) 3,90
5,84 Grm. oder 2,1 pro 1 Kilo Katze.

Dauer des Diabetes 6 Stunden.

Dorpat, den 24. October 1877. 